\title{
Accurate Atom-Dipole Interaction Model for Prediction of Electro- Optical Properties: from van der Waals Aggregates to Covalently Bonded Clusters
}

\author{
Raphael F. Ligórioa, Anna Krawczuk ${ }^{\text {bc }}$ and Leonardo H. R. Dos Santosa* \\ a Departamento de Química, Universidade Federal de Minas Gerais, Av. Pres. Antônio \\ Carlos 6627, 31270-901 Belo Horizonte MG, Brazil. \\ b Institut für Anorganische Chemie, Unisersität Göttingen, Tammannstrasse 4, D-37077 \\ Göttingen, Germany
}

c Faculty of Chemistry, Jagiellonian University in Krakow, Gronostajowa 2, 30-387 Krakow, Poland

*leonardohrs@ufmg.br

\section{Electronic Support Information}

\section{Atomic database for all clusters and molecules}

Table S1: atomic coordinates, dipole moments and polarizabilities of isolated butane, all values in a.u.

\begin{tabular}{ccccccccccccc}
\hline Atom & $\mathbf{x}$ & $\mathbf{y}$ & $\mathbf{z}$ & $\boldsymbol{\mu}_{\mathbf{x}}$ & $\boldsymbol{\mu}_{\mathbf{y}}$ & $\boldsymbol{\mu}_{\mathbf{z}}$ & $\boldsymbol{\alpha}_{\mathbf{x x}}$ & $\boldsymbol{\alpha}_{\mathrm{yy}}$ & $\boldsymbol{\alpha}_{\mathbf{z z}}$ & $\boldsymbol{\alpha}_{\mathrm{xy}}$ & $\boldsymbol{\alpha}_{\mathrm{xz}}$ & $\boldsymbol{\alpha}_{\mathbf{y z}}$ \\
\hline $\mathbf{C}$ & $-0,556$ & 2,462 & 6,342 & 0,04 & $-0,04$ & 0,03 & 6,8 & 6,9 & 5,7 & $-1,9$ & 1,1 & $-1,2$ \\
\hline $\mathbf{H}$ & $-1,951$ & 3,372 & 7,550 & 0,11 & $-0,08$ & $-0,08$ & 3,5 & 2,5 & 2,2 & $-1,6$ & $-1,2$ & 0,9 \\
\hline $\mathbf{H}$ & $-1,516$ & 1,412 & 4,855 & 0,08 & 0,07 & 0,13 & 2,6 & 1,9 & 3,4 & 0,6 & 1,5 & 0,9 \\
\hline $\mathbf{H}$ & 0,657 & 3,893 & 5,496 & $-0,08$ & $-0,12$ & 0,08 & 2,1 & 3,6 & 2,2 & 1,1 & $-0,6$ & $-1,3$ \\
\hline $\mathbf{C}$ & 1,036 & 0,663 & 7,907 & 0,03 & $-0,01$ & $-0,06$ & 7,4 & 7,0 & 5,8 & $-2,9$ & 0,1 & $-0,5$ \\
\hline $\mathbf{H}$ & 1,950 & 1,724 & 9,415 & $-0,07$ & $-0,08$ & $-0,14$ & 1,9 & 2,3 & 3,7 & 0,5 & 1,0 & 1,4 \\
\hline $\mathbf{H}$ & $-0,200$ & $-0,731$ & 8,781 & 0,10 & 0,11 & $-0,09$ & 2,8 & 2,7 & 2,3 & 1,2 & $-1,1$ & $-1,1$ \\
\hline $\mathbf{C}$ & 2,986 & $-0,663$ & 6,435 & $-0,03$ & 0,01 & 0,06 & 7,6 & 6,8 & 5,7 & $-2,9$ & 0,0 & $-0,4$ \\
\hline $\mathbf{C}$ & 4,577 & $-2,462$ & 8,000 & $-0,04$ & 0,04 & $-0,03$ & 6,7 & 6,9 & 5,7 & $-1,9$ & 1,2 & $-1,2$ \\
\hline $\mathbf{H}$ & 5,973 & $-3,372$ & 6,792 & $-0,11$ & 0,08 & 0,08 & 3,5 & 2,5 & 2,2 & $-1,6$ & $-1,2$ & 0,9 \\
\hline $\mathbf{H}$ & 5,538 & $-1,412$ & 9,487 & $-0,08$ & $-0,07$ & $-0,13$ & 2,6 & 1,9 & 3,4 & 0,6 & 1,5 & 0,9 \\
\hline $\mathbf{H}$ & 3,365 & $-3,893$ & 8,846 & 0,08 & 0,12 & $-0,08$ & 2,1 & 3,6 & 2,2 & 1,1 & $-0,6$ & $-1,3$ \\
\hline $\mathbf{H}$ & 2,072 & $-1,724$ & 4,927 & 0,07 & 0,08 & 0,14 & 1,9 & 2,3 & 3,7 & 0,5 & 1,0 & 1,4 \\
\hline $\mathbf{H}$ & 4,222 & 0,731 & 5,561 & $-0,10$ & $-0,11$ & 0,09 & 2,8 & 2,7 & 2,3 & 1,2 & $-1,1$ & $-1,1$ \\
\hline
\end{tabular}


Table S2: atomic coordinates, dipole moments and polarizabilities of the central butane within a three-molecule cluster using QTAIM, all values in a.u.

\begin{tabular}{ccccccccccccc}
\hline Atom & $\mathbf{x}$ & $\mathbf{y}$ & $\mathbf{z}$ & $\boldsymbol{\mu}_{\mathbf{x}}$ & $\boldsymbol{\mu}_{\mathbf{y}}$ & $\boldsymbol{\mu}_{\mathbf{z}}$ & $\boldsymbol{\alpha}_{\mathbf{x x}}$ & $\boldsymbol{\alpha}_{\mathrm{yy}}$ & $\boldsymbol{\alpha}_{\mathrm{zz}}$ & $\boldsymbol{\alpha}_{\mathbf{x y}}$ & $\boldsymbol{\alpha}_{\mathrm{xz}}$ & $\boldsymbol{\alpha}_{\mathrm{yz}}$ \\
\hline $\mathbf{C}$ & $-0,556$ & 2,462 & 6,342 & 0,03 & $-0,04$ & 0,03 & 7,3 & 7,3 & 5,4 & $-2,6$ & 1,1 & $-1,2$ \\
\hline $\mathbf{H}$ & $-1,951$ & 3,372 & 7,550 & 0,10 & $-0,07$ & $-0,07$ & 3,9 & 3,0 & 2,0 & $-2,1$ & $-1,2$ & 0,9 \\
\hline $\mathbf{H}$ & $-1,516$ & 1,412 & 4,855 & 0,08 & 0,07 & 0,13 & 2,5 & 2,0 & 3,3 & 0,5 & 1,4 & 0,9 \\
\hline $\mathbf{H}$ & 0,657 & 3,893 & 5,496 & $-0,08$ & $-0,12$ & 0,08 & 2,1 & 3,5 & 2,1 & 0,9 & $-0,6$ & $-1,2$ \\
\hline $\mathbf{C}$ & 1,036 & 0,663 & 7,907 & 0,03 & $-0,01$ & $-0,06$ & 7,9 & 7,4 & 5,6 & $-3,5$ & 0,1 & $-0,4$ \\
\hline $\mathbf{H}$ & 1,950 & 1,724 & 9,415 & $-0,07$ & $-0,08$ & $-0,14$ & 1,9 & 2,3 & 3,5 & 0,4 & 1,0 & 1,3 \\
\hline $\mathbf{H}$ & $-0,200$ & $-0,731$ & 8,781 & 0,10 & 0,11 & $-0,09$ & 2,7 & 2,8 & 2,3 & 1,0 & $-1,1$ & $-1,1$ \\
\hline $\mathbf{C}$ & 2,986 & $-0,663$ & 6,435 & $-0,03$ & 0,01 & 0,06 & 8,1 & 7,2 & 5,5 & $-3,5$ & 0,0 & $-0,4$ \\
\hline $\mathbf{C}$ & 4,577 & $-2,462$ & 8,000 & $-0,03$ & 0,04 & $-0,03$ & 7,3 & 7,4 & 5,5 & $-2,6$ & 1,1 & $-1,2$ \\
\hline $\mathbf{H}$ & 5,973 & $-3,372$ & 6,792 & $-0,10$ & 0,07 & 0,07 & 3,9 & 3,0 & 2,0 & $-2,2$ & $-1,2$ & 0,9 \\
\hline $\mathbf{H}$ & 5,538 & $-1,412$ & 9,487 & $-0,08$ & $-0,07$ & $-0,13$ & 2,5 & 1,9 & 3,3 & 0,5 & 1,4 & 0,9 \\
\hline $\mathbf{H}$ & 3,365 & $-3,893$ & 8,846 & 0,08 & 0,12 & $-0,08$ & 2,1 & 3,5 & 2,1 & 0,9 & $-0,6$ & $-1,2$ \\
\hline $\mathbf{H}$ & 2,072 & $-1,724$ & 4,927 & 0,07 & 0,08 & 0,14 & 1,9 & 2,3 & 3,5 & 0,4 & 1,0 & 1,3 \\
\hline $\mathbf{H}$ & 4,222 & 0,731 & 5,561 & $-0,10$ & $-0,11$ & 0,09 & 2,7 & 2,8 & 2,3 & 1,0 & $-1,1$ & $-1,1$ \\
\hline
\end{tabular}

Table S3: atomic coordinates, dipole moments and polarizabilities of the central butane within a three-molecule cluster using ADIM, all values in a.u.

\begin{tabular}{ccccccccccccc}
\hline Atom & $\mathbf{x}$ & $\mathbf{y}$ & $\mathbf{z}$ & $\boldsymbol{\mu}_{\mathbf{x}}$ & $\boldsymbol{\mu}_{\mathbf{y}}$ & $\boldsymbol{\mu}_{\mathbf{z}}$ & $\boldsymbol{\alpha}_{\mathbf{x x}}$ & $\boldsymbol{\alpha}_{\mathrm{yy}}$ & $\boldsymbol{\alpha}_{\mathbf{z z}}$ & $\boldsymbol{\alpha}_{\mathrm{xy}}$ & $\boldsymbol{\alpha}_{\mathbf{x z}}$ & $\boldsymbol{\alpha}_{\mathrm{yz}}$ \\
\hline $\mathbf{C}$ & $-0,556$ & 2,462 & 6,342 & 0,03 & $-0,04$ & 0,03 & 7,3 & 7,4 & 5,4 & $-2,6$ & 1,0 & $-1,3$ \\
\hline $\mathbf{H}$ & $-1,951$ & 3,372 & 7,550 & 0,11 & $-0,08$ & $-0,08$ & 4,0 & 3,0 & 2,0 & $-2,1$ & $-1,1$ & 1,1 \\
\hline $\mathbf{H}$ & $-1,516$ & 1,412 & 4,855 & 0,08 & 0,07 & 0,13 & 2,5 & 2,0 & 3,3 & 0,4 & 1,4 & 0,9 \\
\hline $\mathbf{H}$ & 0,657 & 3,893 & 5,496 & $-0,08$ & $-0,12$ & 0,08 & 2,2 & 3,5 & 2,1 & 0,9 & $-0,6$ & $-1,2$ \\
\hline $\mathbf{C}$ & 1,036 & 0,663 & 7,907 & 0,03 & $-0,01$ & $-0,06$ & 7,8 & 7,3 & 5,5 & $-3,4$ & 0,1 & $-0,5$ \\
\hline $\mathbf{H}$ & 1,950 & 1,724 & 9,415 & $-0,07$ & $-0,08$ & $-0,14$ & 1,9 & 2,3 & 3,5 & 0,4 & 1,0 & 1,3 \\
\hline $\mathbf{H}$ & $-0,200$ & $-0,731$ & 8,781 & 0,10 & 0,11 & $-0,09$ & 2,7 & 2,8 & 2,2 & 1,0 & $-1,1$ & $-1,1$ \\
\hline $\mathbf{C}$ & 2,986 & $-0,663$ & 6,435 & $-0,03$ & 0,01 & 0,06 & 8,0 & 7,2 & 5,5 & $-3,4$ & 0,0 & $-0,4$ \\
\hline $\mathbf{C}$ & 4,577 & $-2,462$ & 8,000 & $-0,03$ & 0,04 & $-0,03$ & 7,2 & 7,4 & 5,4 & $-2,7$ & 1,0 & $-1,3$ \\
\hline $\mathbf{H}$ & 5,973 & $-3,372$ & 6,792 & $-0,11$ & 0,08 & 0,08 & 4,0 & 3,0 & 2,0 & $-2,1$ & $-1,1$ & 1,1 \\
\hline $\mathbf{H}$ & 5,538 & $-1,412$ & 9,487 & $-0,08$ & $-0,07$ & $-0,13$ & 2,5 & 2,0 & 3,3 & 0,4 & 1,4 & 0,9 \\
\hline $\mathbf{H}$ & 3,365 & $-3,893$ & 8,846 & 0,08 & 0,12 & $-0,08$ & 2,2 & 3,5 & 2,1 & 0,9 & $-0,6$ & $-1,2$ \\
\hline $\mathbf{H}$ & 2,072 & $-1,724$ & 4,927 & 0,07 & 0,08 & 0,14 & 1,9 & 2,3 & 3,5 & 0,4 & 1,0 & 1,3 \\
\hline $\mathbf{H}$ & 4,222 & 0,731 & 5,561 & $-0,10$ & $-0,11$ & 0,09 & 2,7 & 2,8 & 2,2 & 1,0 & $-1,1$ & $-1,1$ \\
\hline
\end{tabular}


Table S4: atomic coordinates, dipole moments and polarizabilities of isolated benzene, all values in a.u.

\begin{tabular}{|c|c|c|c|c|c|c|c|c|c|c|c|c|}
\hline Atom & $x$ & $y$ & $\mathbf{z}$ & $\mu_{x}$ & $\mu_{y}$ & $\mu_{\mathrm{z}}$ & $\alpha_{x x}$ & $\alpha_{y y}$ & $\alpha_{\mathrm{zz}}$ & $\alpha_{x y}$ & $\alpha_{x z}$ & $\alpha_{y z}$ \\
\hline C & $-0,739$ & 2,477 & 0,123 & 0,01 & $-0,08$ & 0,02 & 8,3 & 8,7 & 8,7 & $-0,1$ & 2,4 & 0,8 \\
\hline $\mathrm{H}$ & $-1,212$ & 4,452 & 0,460 & 0,04 & $-0,11$ & 0,00 & 1,5 & 5,0 & 1,2 & $-1,1$ & $-0,2$ & 0,4 \\
\hline C & 1,157 & 1,606 & 1,735 & $-0,01$ & $-0,04$ & $-0,06$ & 8,3 & 10,0 & 8,1 & $-1,3$ & 1,7 & 0,1 \\
\hline $\mathrm{H}$ & 2,091 & 2,946 & 2,987 & $-0,05$ & $-0,06$ & $-0,07$ & 2,0 & 2,8 & 2,8 & 1,2 & 1,1 & 1,6 \\
\hline C & $-1,849$ & 0,906 & $-1,561$ & 0,06 & $-0,02$ & 0,05 & 7,3 & 10,3 & 7,8 & $-0,2$ & 1,4 & 1,3 \\
\hline $\mathrm{H}$ & $-3,338$ & 1,517 & $-2,844$ & 0,08 & $-0,04$ & 0,07 & 3,4 & 1,6 & 2,8 & $-1,0$ & 1,8 & $-0,9$ \\
\hline C & 0,739 & $-2,477$ & $-0,123$ & $-0,01$ & 0,08 & $-0,02$ & 8,4 & 8,7 & 8,7 & $-0,1$ & 2,4 & 0,8 \\
\hline $\mathrm{H}$ & 1,212 & $-4,452$ & $-0,460$ & $-0,04$ & 0,11 & 0,00 & 1,5 & 5,0 & 1,2 & $-1,1$ & $-0,2$ & 0,4 \\
\hline C & $-1,157$ & $-1,606$ & $-1,735$ & 0,01 & 0,04 & 0,06 & 8,3 & 10,0 & 8,0 & $-1,3$ & 1,6 & 0,1 \\
\hline $\mathrm{H}$ & $-2,091$ & $-2,946$ & $-2,987$ & 0,05 & 0,06 & 0,07 & 2,0 & 2,8 & 2,8 & 1,2 & 1,1 & 1,6 \\
\hline C & 1,849 & $-0,906$ & 1,561 & $-0,06$ & 0,02 & $-0,05$ & 7,3 & 10,3 & 7,8 & $-0,2$ & 1,4 & 1,3 \\
\hline $\mathrm{H}$ & 3,338 & $-1,517$ & 2,844 & $-0,08$ & 0,04 & $-0,07$ & 3,4 & 1,6 & 2,8 & $-1,0$ & 1,8 & $-0,9$ \\
\hline
\end{tabular}

Table S5: atomic coordinates, dipole moments and polarizabilities of the central benzene within a three-molecule cluster using QTAIM, all values in a.u.

\begin{tabular}{|c|c|c|c|c|c|c|c|c|c|c|c|c|}
\hline Atom & $\mathrm{x}$ & $y$ & $\mathbf{z}$ & $\mu_{\mathrm{x}}$ & $\mu_{\mathrm{y}}$ & $\mu_{\mathrm{z}}$ & $\alpha_{x x}$ & $\alpha_{y y}$ & $\alpha_{\mathrm{zz}}$ & $\alpha_{\mathrm{xy}}$ & $\alpha_{x z}$ & $\alpha_{y z}$ \\
\hline C & $-0,739$ & 2,477 & 0,123 & 0,02 & $-0,08$ & 0,02 & 7,5 & 9,7 & 9,0 & 0,3 & 2,3 & 2,4 \\
\hline $\mathrm{H}$ & $-1,212$ & 4,452 & 0,460 & 0,04 & $-0,09$ & 0,02 & 1,2 & 5,4 & 1,8 & $-1,0$ & $-0,3$ & 1,2 \\
\hline C & 1,157 & 1,606 & 1,735 & 0,00 & $-0,03$ & $-0,06$ & 7,3 & 11,3 & 7,9 & $-1,3$ & 1,4 & 1,6 \\
\hline $\mathrm{H}$ & 2,091 & 2,946 & 2,987 & $-0,05$ & $-0,04$ & $-0,05$ & 1,8 & 3,4 & 3,0 & 0,9 & 0,8 & 2,3 \\
\hline C & $-1,849$ & 0,906 & $-1,561$ & 0,07 & $-0,03$ & 0,06 & 6,9 & 11,9 & 8,2 & $-0,1$ & 1,4 & 2,7 \\
\hline $\mathrm{H}$ & $-3,338$ & 1,517 & $-2,844$ & 0,09 & $-0,05$ & 0,07 & 3,3 & 1,7 & 2,7 & $-0,9$ & 1,7 & $-0,7$ \\
\hline C & 0,739 & $-2,477$ & $-0,123$ & $-0,02$ & 0,08 & $-0,02$ & 7,5 & 9,7 & 9,0 & 0,2 & 2,3 & 2,4 \\
\hline $\mathrm{H}$ & 1,212 & $-4,452$ & $-0,460$ & $-0,04$ & 0,09 & $-0,02$ & 1,2 & 5,4 & 1,8 & $-1,0$ & $-0,3$ & 1,2 \\
\hline C & $-1,157$ & $-1,606$ & $-1,735$ & 0,00 & 0,03 & 0,06 & 7,3 & 11,3 & 7,9 & $-1,3$ & 1,4 & 1,6 \\
\hline $\mathrm{H}$ & $-2,091$ & $-2,946$ & $-2,987$ & 0,05 & 0,04 & 0,05 & 1,8 & 3,4 & 3,0 & 0,9 & 0,8 & 2,3 \\
\hline C & 1,849 & $-0,906$ & 1,561 & $-0,07$ & 0,03 & $-0,06$ & 6,9 & 11,8 & 8,2 & $-0,1$ & 1,4 & 2,7 \\
\hline $\mathrm{H}$ & 3,338 & $-1,517$ & 2,844 & $-0,09$ & 0,05 & $-0,07$ & 3,3 & 1,7 & 2,7 & $-0,9$ & 1,7 & $-0,7$ \\
\hline
\end{tabular}


Table S6: atomic coordinates, dipole moments and polarizabilities of the central benzene within a three-molecule cluster using ADIM, all values in a.u.

\begin{tabular}{ccccccccccccc}
\hline Atom & $\mathbf{x}$ & $\mathbf{y}$ & $\mathbf{z}$ & $\boldsymbol{\mu}_{\mathbf{x}}$ & $\boldsymbol{\mu}_{\mathrm{y}}$ & $\boldsymbol{\mu}_{\mathrm{z}}$ & $\boldsymbol{\alpha}_{\mathrm{xx}}$ & $\boldsymbol{\alpha}_{\mathrm{yy}}$ & $\boldsymbol{\alpha}_{\mathrm{zz}}$ & $\boldsymbol{\alpha}_{\mathrm{xy}}$ & $\boldsymbol{\alpha}_{\mathrm{xz}}$ & $\boldsymbol{\alpha}_{\mathrm{yz}}$ \\
\hline $\mathbf{C}$ & $-0,739$ & 2,477 & 0,123 & 0,01 & $-0,09$ & 0,01 & 7,4 & 9,4 & 9,0 & 0,2 & 2,1 & 2,4 \\
\hline H & $-1,212$ & 4,452 & 0,460 & 0,04 & $-0,11$ & 0,00 & 1,2 & 5,1 & 1,5 & $-0,7$ & $-0,5$ & 0,7 \\
\hline $\mathbf{C}$ & 1,157 & 1,606 & 1,735 & $-0,01$ & $-0,05$ & $-0,06$ & 7,3 & 10,9 & 7,9 & $-1,2$ & 1,0 & 1,6 \\
\hline H & 2,091 & 2,946 & 2,987 & $-0,05$ & $-0,07$ & $-0,08$ & 1,6 & 3,1 & 2,9 & 0,7 & 1,1 & 2,2 \\
\hline $\mathbf{C}$ & $-1,849$ & 0,906 & $-1,561$ & 0,06 & $-0,02$ & 0,05 & 6,7 & 11,3 & 7,9 & 0,0 & 1,2 & 2,5 \\
\hline H & $-3,338$ & 1,517 & $-2,844$ & 0,08 & $-0,04$ & 0,07 & 3,2 & 1,6 & 2,7 & $-0,9$ & 1,7 & $-0,7$ \\
\hline C & 0,739 & $-2,477$ & $-0,123$ & $-0,01$ & 0,09 & $-0,01$ & 7,4 & 9,4 & 9,0 & 0,2 & 2,1 & 2,4 \\
\hline H & 1,212 & $-4,452$ & $-0,460$ & $-0,04$ & 0,11 & 0,00 & 1,2 & 5,1 & 1,5 & $-0,7$ & $-0,5$ & 0,7 \\
\hline C & $-1,157$ & $-1,606$ & $-1,735$ & 0,01 & 0,05 & 0,06 & 7,3 & 10,9 & 7,9 & $-1,2$ & 1,0 & 1,5 \\
\hline H & $-2,091$ & $-2,946$ & $-2,987$ & 0,05 & 0,07 & 0,08 & 1,6 & 3,1 & 2,9 & 0,7 & 1,1 & 2,2 \\
\hline C & 1,849 & $-0,906$ & 1,561 & $-0,06$ & 0,02 & $-0,05$ & 6,7 & 11,3 & 7,9 & 0,0 & 1,2 & 2,5 \\
\hline H & 3,338 & $-1,517$ & 2,844 & $-0,08$ & 0,04 & $-0,07$ & 3,2 & 1,6 & 2,7 & $-0,9$ & 1,7 & $-0,7$ \\
\hline
\end{tabular}

Table S7: atomic coordinates, dipole moments and polarizabilities of isolated pyridazine, all values in a.u.

\begin{tabular}{ccccccccccccc}
\hline Atom & $\mathbf{x}$ & $\mathbf{y}$ & $\mathbf{z}$ & $\boldsymbol{\mu}_{\mathrm{x}}$ & $\boldsymbol{\mu}_{\mathrm{y}}$ & $\boldsymbol{\mu}_{\mathrm{z}}$ & $\boldsymbol{\alpha}_{\mathrm{xx}}$ & $\boldsymbol{\alpha}_{\mathbf{y y}}$ & $\boldsymbol{\alpha}_{\mathrm{zz}}$ & $\boldsymbol{\alpha}_{\mathrm{xy}}$ & $\boldsymbol{\alpha}_{\mathrm{xz}}$ & $\boldsymbol{\alpha}_{\mathrm{yz}}$ \\
\hline $\mathbf{N}$ & $-0,441$ & 17,764 & 7,057 & 0,35 & 0,36 & $-0,84$ & 7,3 & 12,3 & 9,8 & 2,5 & $-0,9$ & 0,0 \\
\hline $\mathbf{N}$ & $-0,982$ & 15,481 & 5,629 & 0,36 & 0,56 & $-0,69$ & 6,7 & 9,9 & 12,5 & 1,3 & $-1,1$ & 0,6 \\
\hline $\mathbf{C}$ & $-0,389$ & 15,757 & 3,211 & 0,03 & 0,07 & $-0,01$ & 5,1 & 6,9 & 6,6 & 1,4 & $-0,8$ & $-0,7$ \\
\hline $\mathbf{H}$ & $-0,886$ & 14,172 & 1,996 & 0,02 & 0,07 & 0,06 & 1,4 & 3,5 & 2,4 & 0,6 & 0,6 & 1,8 \\
\hline $\mathbf{C}$ & 0,739 & 17,711 & 2,038 & 0,00 & 0,02 & 0,04 & 6,3 & 9,0 & 8,1 & 1,2 & $-0,3$ & 0,6 \\
\hline $\mathbf{H}$ & 1,195 & 17,655 & 0,032 & $-0,02$ & 0,01 & 0,07 & 1,3 & 1,1 & 4,5 & $-0,1$ & $-0,7$ & 0,2 \\
\hline $\mathbf{C}$ & 1,278 & 19,751 & 3,482 & $-0,01$ & $-0,05$ & $-0,02$ & 6,2 & 8,5 & 9,0 & 1,0 & $-0,4$ & 1,0 \\
\hline $\mathbf{H}$ & 2,238 & 21,375 & 2,660 & $-0,04$ & $-0,07$ & 0,03 & 2,0 & 3,6 & 1,7 & 1,4 & $-0,6$ & $-1,2$ \\
\hline $\mathbf{C}$ & 0,599 & 19,762 & 6,015 & $-0,01$ & $-0,03$ & $-0,03$ & 5,0 & 6,3 & 8,0 & 0,7 & $-0,9$ & 0,1 \\
\hline $\mathbf{H}$ & 0,933 & 21,444 & 7,152 & $-0,01$ & $-0,06$ & $-0,03$ & 1,3 & 3,5 & 2,2 & 0,5 & 0,4 & 1,7 \\
\hline
\end{tabular}

Table S8: atomic coordinates, dipole moments and polarizabilities of the central pyridazine within a three-molecule cluster using QTAIM, all values in a.u.

\begin{tabular}{ccccccccccccc}
\hline Atom & $\mathbf{x}$ & $\mathbf{y}$ & $\mathbf{z}$ & $\boldsymbol{\mu}_{\mathbf{x}}$ & $\boldsymbol{\mu}_{\mathbf{y}}$ & $\boldsymbol{\mu}_{\mathbf{z}}$ & $\boldsymbol{\alpha}_{\mathbf{x x}}$ & $\boldsymbol{\alpha}_{\mathbf{y y}}$ & $\boldsymbol{\alpha}_{\mathrm{zz}}$ & $\boldsymbol{\alpha}_{\mathrm{xy}}$ & $\boldsymbol{\alpha}_{\mathrm{xz}}$ & $\boldsymbol{\alpha}_{\mathrm{yz}}$ \\
\hline $\mathbf{N}$ & $-0,441$ & 17,764 & 7,057 & 0,36 & 0,38 & $-0,86$ & 7,2 & 14,5 & 9,3 & 3,3 & $-0,9$ & $-0,2$ \\
\hline $\mathbf{N}$ & $-0,982$ & 15,481 & 5,629 & 0,37 & 0,55 & $-0,70$ & 6,5 & 11,9 & 11,2 & 2,3 & $-0,9$ & 0,9 \\
\hline $\mathbf{C}$ & $-0,389$ & 15,757 & 3,211 & 0,05 & 0,09 & $-0,02$ & 5,0 & 8,0 & 6,0 & 2,0 & $-0,8$ & $-0,9$ \\
\hline $\mathbf{H}$ & $-0,886$ & 14,172 & 1,996 & 0,03 & 0,07 & 0,06 & 1,5 & 4,1 & 2,1 & 1,1 & 0,5 & 1,6 \\
\hline $\mathbf{C}$ & 0,739 & 17,711 & 2,038 & 0,01 & 0,03 & 0,03 & 6,1 & 10,4 & 7,7 & 1,8 & $-0,3$ & 0,7 \\
\hline $\mathbf{H}$ & 1,195 & 17,655 & 0,032 & $-0,01$ & 0,01 & 0,07 & 1,3 & 1,1 & 4,3 & 0,0 & $-0,7$ & 0,2 \\
\hline $\mathbf{C}$ & 1,278 & 19,751 & 3,482 & 0,01 & $-0,03$ & $-0,04$ & 5,8 & 10,1 & 7,9 & 1,7 & $-0,2$ & 1,2 \\
\hline $\mathbf{H}$ & 2,238 & 21,375 & 2,660 & $-0,02$ & $-0,05$ & 0,02 & 1,7 & 4,3 & 1,3 & 1,5 & $-0,4$ & $-0,8$ \\
\hline $\mathbf{C}$ & 0,599 & 19,762 & 6,015 & 0,00 & $-0,02$ & $-0,05$ & 4,8 & 7,4 & 7,1 & 1,2 & $-0,8$ & $-0,1$ \\
\hline $\mathbf{H}$ & 0,933 & 21,444 & 7,152 & 0,00 & $-0,04$ & $-0,04$ & 1,3 & 4,2 & 1,9 & 0,9 & 0,3 & 1,5 \\
\hline
\end{tabular}


Table S9: atomic coordinates, dipole moments and polarizabilities of the central pyridazine within a three-molecule cluster using ADIM, all values in a.u.

\begin{tabular}{ccccccccccccc}
\hline Atom & $\mathbf{x}$ & $\mathbf{y}$ & $\mathbf{z}$ & $\boldsymbol{\mu}_{\mathrm{x}}$ & $\boldsymbol{\mu}_{\mathrm{y}}$ & $\boldsymbol{\mu}_{\mathrm{z}}$ & $\boldsymbol{\alpha}_{\mathrm{xx}}$ & $\boldsymbol{\alpha}_{\mathrm{yy}}$ & $\boldsymbol{\alpha}_{\mathrm{zz}}$ & $\boldsymbol{\alpha}_{\mathrm{xy}}$ & $\boldsymbol{\alpha}_{\mathrm{xz}}$ & $\boldsymbol{\alpha}_{\mathrm{yz}}$ \\
\hline $\mathbf{N}$ & $-0,441$ & 17,764 & 7,057 & 0,36 & 0,37 & $-0,85$ & 7,0 & 13,9 & 9,0 & 2,9 & $-0,9$ & $-0,1$ \\
\hline $\mathbf{N}$ & $-0,982$ & 15,481 & 5,629 & 0,38 & 0,59 & $-0,71$ & 6,4 & 11,4 & 11,1 & 1,9 & $-1,0$ & 0,9 \\
\hline $\mathbf{C}$ & $-0,389$ & 15,757 & 3,211 & 0,04 & 0,09 & $-0,03$ & 5,0 & 7,9 & 6,0 & 1,7 & $-0,9$ & $-0,9$ \\
\hline $\mathbf{H}$ & $-0,886$ & 14,172 & 1,996 & 0,02 & 0,07 & 0,05 & 1,4 & 4,0 & 1,9 & 0,9 & 0,4 & 2,0 \\
\hline $\mathbf{C}$ & 0,739 & 17,711 & 2,038 & 0,02 & 0,03 & 0,02 & 6,0 & 10,1 & 7,5 & 1,5 & $-0,3$ & 0,8 \\
\hline $\mathbf{H}$ & 1,195 & 17,655 & 0,032 & $-0,01$ & 0,01 & 0,07 & 1,3 & 1,2 & 4,3 & 0,0 & $-0,7$ & 0,2 \\
\hline $\mathbf{C}$ & 1,278 & 19,751 & 3,482 & 0,02 & $-0,04$ & $-0,05$ & 5,8 & 10,1 & 8,0 & 1,4 & $-0,3$ & 1,4 \\
\hline $\mathbf{H}$ & 2,238 & 21,375 & 2,660 & $-0,01$ & $-0,03$ & 0,01 & 1,8 & 4,6 & 1,3 & 1,4 & $-0,3$ & $-1,4$ \\
\hline $\mathbf{C}$ & 0,599 & 19,762 & 6,015 & 0,01 & $-0,03$ & $-0,05$ & 4,7 & 7,3 & 7,1 & 1,0 & $-0,9$ & $-0,1$ \\
\hline $\mathbf{H}$ & 0,933 & 21,444 & 7,152 & $-0,01$ & $-0,08$ & $-0,04$ & 1,2 & 4,1 & 1,7 & 0,8 & 0,2 & 1,9 \\
\hline
\end{tabular}

Table S10: atomic coordinates, dipole moments and polarizabilities of isolated 1,3nitrophenol, all values in a.u.

\begin{tabular}{ccccccccccccc}
\hline Atom & $\mathbf{x}$ & $\mathbf{y}$ & $\mathbf{z}$ & $\boldsymbol{\mu}_{\mathbf{x}}$ & $\boldsymbol{\mu}_{\mathbf{y}}$ & $\boldsymbol{\mu}_{\mathbf{z}}$ & $\boldsymbol{\alpha}_{\mathbf{x x}}$ & $\boldsymbol{\alpha}_{\mathrm{yy}}$ & $\boldsymbol{\alpha}_{\mathrm{zz}}$ & $\boldsymbol{\alpha}_{\mathrm{xy}}$ & $\boldsymbol{\alpha}_{\mathrm{xz}}$ & $\boldsymbol{\alpha}_{\mathrm{yz}}$ \\
\hline $\mathbf{C}$ & 13,218 & 1,961 & 13,674 & 0,07 & 0,05 & $-0,29$ & 8,7 & 6,5 & 10,6 & 2,9 & 0,2 & 0,3 \\
\hline $\mathbf{C}$ & 11,833 & 0,950 & 11,692 & $-0,08$ & $-0,07$ & $-0,16$ & 6,6 & 5,9 & 13,2 & 0,9 & 0,5 & 0,6 \\
\hline $\mathbf{C}$ & 12,660 & 1,424 & 9,238 & $-0,11$ & $-0,09$ & $-0,18$ & 7,8 & 5,7 & 9,8 & 2,6 & $-1,7$ & $-0,9$ \\
\hline $\mathbf{C}$ & 14,815 & 2,908 & 8,833 & $-0,04$ & $-0,03$ & 0,03 & 9,3 & 7,6 & 9,6 & 2,2 & 0,6 & 0,5 \\
\hline $\mathbf{C}$ & 16,147 & 3,896 & 10,880 & $-0,02$ & $-0,01$ & $-0,06$ & 7,7 & 6,7 & 11,7 & 1,4 & 0,0 & 0,2 \\
\hline $\mathbf{C}$ & 15,368 & 3,426 & 13,346 & 0,11 & 0,07 & $-0,07$ & 8,8 & 7,0 & 9,9 & 2,2 & $-1,8$ & $-1,1$ \\
\hline $\mathbf{N}$ & 12,334 & 1,468 & 16,242 & 0,26 & 0,14 & $-0,77$ & 6,9 & 4,5 & 15,1 & 3,1 & $-2,4$ & $-1,1$ \\
\hline $\mathbf{0}$ & 13,540 & 2,368 & 18,016 & $-0,50$ & $-0,37$ & $-0,67$ & 5,6 & 5,2 & 10,1 & 0,8 & 2,6 & 2,1 \\
\hline $\mathbf{O}$ & 10,457 & 0,139 & 16,537 & 0,74 & 0,52 & $-0,07$ & 9,0 & 6,7 & 5,0 & 3,1 & $-1,8$ & $-1,3$ \\
\hline $\mathbf{0}$ & 11,303 & 0,420 & 7,319 & 0,63 & 0,52 & 0,00 & 6,6 & 6,0 & 10,9 & 1,1 & 3,6 & 2,6 \\
\hline $\mathbf{H}$ & 10,154 & $-0,176$ & 12,077 & 0,02 & 0,01 & 0,02 & 2,6 & 1,7 & 0,8 & 1,2 & $-0,1$ & $-0,1$ \\
\hline $\mathbf{H}$ & 15,458 & 3,294 & 6,917 & $-0,02$ & $-0,01$ & 0,09 & 1,5 & 1,3 & 4,6 & 0,2 & $-1,2$ & $-0,7$ \\
\hline $\mathbf{H}$ & 17,819 & 5,051 & 10,557 & $-0,07$ & $-0,05$ & 0,02 & 3,7 & 2,4 & 1,2 & 1,7 & $-0,6$ & $-0,4$ \\
\hline $\mathbf{H}$ & 16,446 & 4,196 & 14,922 & $-0,03$ & $-0,02$ & $-0,02$ & 2,0 & 1,5 & 2,3 & 0,7 & 1,2 & 0,9 \\
\hline $\mathbf{H}$ & 11,959 & 1,055 & 5,680 & 0,02 & 0,02 & $-0,06$ & 0,3 & 0,3 & 1,7 & 0,0 & $-0,2$ & $-0,3$ \\
\hline
\end{tabular}


Table S11: atomic coordinates, dipole moments and polarizabilities of the central 1,3nitrophenol within a three-molecule cluster using QTAIM, all values in a.u.

\begin{tabular}{ccccccccccccc}
\hline Atom & $\mathbf{x}$ & $\mathbf{y}$ & $\mathbf{z}$ & $\boldsymbol{\mu}_{\mathbf{x}}$ & $\boldsymbol{\mu}_{\mathbf{y}}$ & $\boldsymbol{\mu}_{\mathbf{z}}$ & $\boldsymbol{\alpha}_{\mathbf{x x}}$ & $\boldsymbol{\alpha}_{\mathrm{yy}}$ & $\boldsymbol{\alpha}_{\mathrm{zz}}$ & $\boldsymbol{\alpha}_{\mathrm{xy}}$ & $\boldsymbol{\alpha}_{\mathrm{xz}}$ & $\boldsymbol{\alpha}_{\mathrm{yz}}$ \\
\hline $\mathbf{C}$ & 13,218 & 1,961 & 13,674 & 0,07 & 0,04 & $-0,37$ & 8,4 & 6,3 & 13,8 & 2,7 & 0,1 & 0,3 \\
\hline $\mathbf{C}$ & 11,833 & 0,950 & 11,692 & $-0,09$ & $-0,08$ & $-0,24$ & 6,3 & 5,7 & 16,4 & 0,8 & 0,5 & 0,7 \\
\hline $\mathbf{C}$ & 12,660 & 1,424 & 9,238 & $-0,11$ & $-0,09$ & $-0,24$ & 7,0 & 5,2 & 12,2 & 2,2 & $-1,9$ & $-1,1$ \\
\hline $\mathbf{C}$ & 14,815 & 2,908 & 8,833 & $-0,06$ & $-0,04$ & $-0,04$ & 8,4 & 7,0 & 11,9 & 1,9 & 1,1 & 0,9 \\
\hline $\mathbf{C}$ & 16,147 & 3,896 & 10,880 & $-0,03$ & $-0,02$ & $-0,12$ & 7,5 & 6,5 & 14,1 & 1,3 & 0,3 & 0,4 \\
\hline $\mathbf{C}$ & 15,368 & 3,426 & 13,346 & 0,12 & 0,08 & $-0,11$ & 8,4 & 6,8 & 11,9 & 2,0 & $-2,1$ & $-1,3$ \\
\hline $\mathbf{N}$ & 12,334 & 1,468 & 16,242 & 0,23 & 0,12 & $-0,91$ & 6,2 & 4,0 & 19,7 & 2,7 & $-2,5$ & $-1,1$ \\
\hline $\mathbf{0}$ & 13,540 & 2,368 & 18,016 & $-0,52$ & $-0,38$ & $-0,83$ & 5,0 & 4,7 & 16,2 & 0,7 & 2,6 & 1,9 \\
\hline $\mathbf{0}$ & 10,457 & 0,139 & 16,537 & 0,69 & 0,49 & $-0,08$ & 8,1 & 6,0 & 5,5 & 2,7 & $-1,2$ & $-0,9$ \\
\hline $\mathbf{0}$ & 11,303 & 0,420 & 7,319 & 0,63 & 0,53 & $-0,13$ & 6,0 & 5,5 & 14,6 & 0,9 & 3,1 & 2,2 \\
\hline $\mathbf{H}$ & 10,154 & $-0,176$ & 12,077 & 0,02 & 0,01 & 0,01 & 2,5 & 1,6 & 1,0 & 1,1 & $-0,2$ & $-0,1$ \\
\hline $\mathbf{H}$ & 15,458 & 3,294 & 6,917 & $-0,03$ & $-0,02$ & 0,04 & 1,5 & 1,1 & 5,4 & 0,3 & $-0,7$ & $-0,4$ \\
\hline $\mathbf{H}$ & 17,819 & 5,051 & 10,557 & $-0,07$ & $-0,05$ & 0,02 & 3,6 & 2,3 & 1,3 & 1,7 & $-0,6$ & $-0,4$ \\
\hline $\mathbf{H}$ & 16,446 & 4,196 & 14,922 & $-0,03$ & $-0,02$ & $-0,03$ & 1,9 & 1,4 & 2,7 & 0,7 & 1,3 & 0,9 \\
\hline $\mathbf{H}$ & 11,959 & 1,055 & 5,680 & 0,05 & 0,04 & $-0,12$ & 0,2 & 0,2 & 2,0 & 0,1 & $-0,5$ & $-0,4$ \\
\hline
\end{tabular}

Table S12: atomic coordinates, dipole moments and polarizabilities of the central 1,3nitrophenol within a three-molecule cluster using ADIM, all values in a.u.

\begin{tabular}{ccccccccccccc}
\hline Atom & $\mathbf{x}$ & $\mathbf{y}$ & $\mathbf{z}$ & $\boldsymbol{\mu}_{\mathbf{x}}$ & $\boldsymbol{\mu}_{\mathbf{y}}$ & $\boldsymbol{\mu}_{\mathbf{z}}$ & $\boldsymbol{\alpha}_{\mathrm{xx}}$ & $\boldsymbol{\alpha}_{\mathrm{yy}}$ & $\boldsymbol{\alpha}_{\mathrm{zz}}$ & $\boldsymbol{\alpha}_{\mathrm{xy}}$ & $\boldsymbol{\alpha}_{\mathrm{xz}}$ & $\boldsymbol{\alpha}_{\mathrm{yz}}$ \\
\hline $\mathbf{C}$ & 13,218 & 1,961 & 13,674 & 0,07 & 0,04 & $-0,34$ & 8,1 & 6,1 & 12,4 & 2,6 & 0,3 & 0,4 \\
\hline $\mathbf{C}$ & 11,833 & 0,950 & 11,692 & $-0,09$ & $-0,07$ & $-0,21$ & 6,2 & 5,6 & 15,3 & 0,8 & 0,5 & 0,6 \\
\hline $\mathbf{C}$ & 12,660 & 1,424 & 9,238 & $-0,09$ & $-0,07$ & $-0,26$ & 7,0 & 5,2 & 12,1 & 2,2 & $-2,3$ & $-0,8$ \\
\hline $\mathbf{C}$ & 14,815 & 2,908 & 8,833 & $-0,07$ & $-0,05$ & $-0,07$ & 8,6 & 7,1 & 11,9 & 2,0 & 1,3 & 0,9 \\
\hline $\mathbf{C}$ & 16,147 & 3,896 & 10,880 & $-0,03$ & $-0,02$ & $-0,12$ & 7,4 & 6,4 & 13,4 & 1,3 & 0,2 & 0,4 \\
\hline $\mathbf{C}$ & 15,368 & 3,426 & 13,346 & 0,11 & 0,07 & $-0,11$ & 8,3 & 6,6 & 11,4 & 2,0 & $-2,2$ & $-1,1$ \\
\hline $\mathbf{N}$ & 12,334 & 1,468 & 16,242 & 0,26 & 0,14 & $-0,89$ & 6,1 & 4,0 & 19,2 & 2,6 & $-3,0$ & $-0,8$ \\
\hline $\mathbf{0}$ & 13,540 & 2,368 & 18,016 & $-0,53$ & $-0,39$ & $-0,82$ & 4,6 & 4,4 & 14,1 & 0,5 & 3,5 & 1,4 \\
\hline $\mathbf{0}$ & 10,457 & 0,139 & 16,537 & 0,70 & 0,49 & $-0,08$ & 8,2 & 6,1 & 6,1 & 2,7 & $-1,5$ & $-0,9$ \\
\hline $\mathbf{0}$ & 11,303 & 0,420 & 7,319 & 0,63 & 0,52 & $-0,06$ & 5,4 & 5,2 & 13,7 & 0,6 & 4,0 & 1,6 \\
\hline $\mathbf{H}$ & 10,154 & $-0,176$ & 12,077 & 0,02 & 0,01 & 0,02 & 2,5 & 1,6 & 1,0 & 1,1 & $-0,2$ & $-0,1$ \\
\hline $\mathbf{H}$ & 15,458 & 3,294 & 6,917 & $-0,01$ & 0,00 & 0,02 & 1,2 & 1,1 & 6,0 & 0,1 & $-1,4$ & $-0,2$ \\
\hline $\mathbf{H}$ & 17,819 & 5,051 & 10,557 & $-0,07$ & $-0,05$ & 0,01 & 3,6 & 2,3 & 1,3 & 1,7 & $-0,5$ & $-0,4$ \\
\hline $\mathbf{H}$ & 16,446 & 4,196 & 14,922 & $-0,03$ & $-0,02$ & $-0,02$ & 1,8 & 1,4 & 2,6 & 0,6 & 1,3 & 0,8 \\
\hline $\mathbf{H}$ & 11,959 & 1,055 & 5,680 & 0,02 & 0,03 & $-0,09$ & 0,2 & 0,3 & 2,9 & 0,0 & $-0,4$ & $-0,4$
\end{tabular}


Table S13: atomic coordinates, dipole moments and polarizabilities of isolated 1,4nitrophenol, all values in a.u.

\begin{tabular}{ccccccccccccc}
\hline Atom & $\mathbf{x}$ & $\mathbf{y}$ & $\mathbf{z}$ & $\boldsymbol{\mu}_{\mathbf{x}}$ & $\boldsymbol{\mu}_{\mathbf{y}}$ & $\boldsymbol{\mu}_{\mathrm{z}}$ & $\boldsymbol{\alpha}_{\mathrm{xx}}$ & $\boldsymbol{\alpha}_{\mathrm{yy}}$ & $\boldsymbol{\alpha}_{\mathrm{zz}}$ & $\boldsymbol{\alpha}_{\mathrm{xy}}$ & $\boldsymbol{\alpha}_{\mathrm{xz}}$ & $\boldsymbol{\alpha}_{\mathrm{yz}}$ \\
\hline $\mathbf{N}$ & 0,481 & 4,298 & 10,038 & 0,46 & $-0,40$ & 0,63 & 9,7 & 7,7 & 9,9 & $-0,8$ & 5,2 & $-5,1$ \\
\hline $\mathbf{O}$ & 6,088 & $-0,236$ & 17,582 & 0,17 & 0,67 & $-0,44$ & 8,0 & 6,3 & 10,2 & $-2,4$ & 4,1 & $-2,7$ \\
\hline $\mathbf{O}$ & 1,318 & 6,173 & 8,948 & $-0,38$ & $-0,76$ & 0,41 & 4,6 & 9,6 & 7,1 & 1,1 & $-1,0$ & $-3,5$ \\
\hline $\mathbf{O}$ & $-1,582$ & 3,420 & 9,533 & 0,82 & 0,39 & 0,17 & 10,8 & 4,8 & 5,3 & 1,7 & 2,4 & 0,8 \\
\hline $\mathbf{C}$ & 2,015 & 3,129 & 11,978 & 0,19 & $-0,16$ & 0,24 & 9,6 & 8,4 & 8,2 & 0,9 & 2,2 & $-2,6$ \\
\hline $\mathbf{C}$ & 4,350 & 4,100 & 12,554 & 0,15 & 0,04 & 0,06 & 10,0 & 6,2 & 8,1 & 0,0 & 3,2 & $-1,2$ \\
\hline $\mathbf{H}$ & 5,082 & 5,723 & 11,523 & $-0,03$ & $-0,03$ & 0,00 & 1,5 & 2,6 & 1,4 & 1,0 & $-0,6$ & $-0,9$ \\
\hline $\mathbf{C}$ & 5,744 & 3,010 & 14,431 & $-0,05$ & $-0,10$ & 0,05 & 8,1 & 9,0 & 9,6 & $-0,3$ & 1,7 & $-2,9$ \\
\hline $\mathbf{H}$ & 7,587 & 3,782 & 14,926 & $-0,09$ & $-0,03$ & $-0,03$ & 4,3 & 1,6 & 1,4 & 1,2 & 0,9 & 0,4 \\
\hline $\mathbf{C}$ & 4,811 & 0,924 & 15,703 & 0,08 & $-0,14$ & 0,18 & 8,2 & 7,3 & 6,5 & 1,8 & 1,3 & $-2,0$ \\
\hline $\mathbf{C}$ & 2,492 & $-0,064$ & 15,054 & 0,09 & 0,01 & 0,05 & 10,2 & 7,4 & 8,8 & 0,0 & 2,9 & $-1,5$ \\
\hline $\mathbf{H}$ & 1,789 & $-1,735$ & 16,028 & 0,03 & 0,06 & $-0,03$ & 1,4 & 3,5 & 1,9 & 1,0 & $-0,6$ & $-1,4$ \\
\hline $\mathbf{C}$ & 1,071 & 1,026 & 13,207 & $-0,03$ & $-0,16$ & 0,12 & 6,9 & 8,6 & 8,9 & $-0,4$ & 1,6 & $-3,4$ \\
\hline $\mathbf{H}$ & $-0,771$ & 0,257 & 12,708 & 0,03 & 0,03 & $-0,01$ & 3,0 & 1,5 & 1,0 & 1,1 & 0,3 & 0,2 \\
\hline $\mathbf{H}$ & 7,670 & 0,718 & 17,910 & 0,06 & 0,03 & 0,02 & 1,5 & 0,3 & 0,5 & 0,4 & 0,5 & 0,2 \\
\hline
\end{tabular}

Table S14: atomic coordinates, dipole moments and polarizabilities of the central 1,4nitrophenol within a three-molecule cluster using QTAIM, all values in a.u.

\begin{tabular}{ccccccccccccc}
\hline Atom & $\mathbf{X}$ & $\mathbf{y}$ & $\mathbf{z}$ & $\boldsymbol{\mu}_{\mathbf{x}}$ & $\boldsymbol{\mu}_{\mathbf{y}}$ & $\boldsymbol{\mu}_{\mathbf{z}}$ & $\boldsymbol{\alpha}_{\mathbf{x x}}$ & $\boldsymbol{\alpha}_{\mathrm{yy}}$ & $\boldsymbol{\alpha}_{\mathbf{z z}}$ & $\boldsymbol{\alpha}_{\mathbf{x y}}$ & $\boldsymbol{\alpha}_{\mathbf{x z}}$ & $\boldsymbol{\alpha}_{\mathbf{y z}}$ \\
\hline $\mathbf{N}$ & 0,481 & 4,298 & 10,038 & 0,49 & $-0,48$ & 0,72 & 10,9 & 7,3 & 12,8 & $-2,2$ & 7,9 & $-6,1$ \\
\hline $\mathbf{O}$ & 6,088 & $-0,236$ & 17,582 & 0,28 & 0,71 & $-0,37$ & 9,6 & 6,7 & 11,9 & $-1,4$ & 6,3 & $-2,2$ \\
\hline $\mathbf{O}$ & 1,318 & 6,173 & 8,948 & $-0,35$ & $-0,83$ & 0,51 & 6,0 & 9,0 & 8,9 & $-0,5$ & 1,3 & $-4,7$ \\
\hline $\mathbf{O}$ & $-1,582$ & 3,420 & 9,533 & 0,80 & 0,37 & 0,20 & 10,5 & 4,2 & 6,5 & 1,4 & 3,5 & 0,3 \\
\hline $\mathbf{C}$ & 2,015 & 3,129 & 11,978 & 0,24 & $-0,18$ & 0,30 & 11,2 & 8,3 & 10,5 & 0,3 & 4,3 & $-3,4$ \\
\hline $\mathbf{C}$ & 4,350 & 4,100 & 12,554 & 0,22 & 0,02 & 0,12 & 11,4 & 5,8 & 9,6 & $-0,1$ & 4,9 & $-1,3$ \\
\hline $\mathbf{H}$ & 5,082 & 5,723 & 11,523 & $-0,03$ & $-0,03$ & 0,01 & 1,5 & 2,5 & 1,6 & 0,9 & $-0,5$ & $-0,9$ \\
\hline $\mathbf{C}$ & 5,744 & 3,010 & 14,431 & $-0,01$ & $-0,12$ & 0,13 & 8,5 & 8,2 & 11,2 & $-0,5$ & 3,3 & $-3,2$ \\
\hline $\mathbf{H}$ & 7,587 & 3,782 & 14,926 & $-0,07$ & $-0,02$ & 0,00 & 4,1 & 1,3 & 2,2 & 1,2 & 1,2 & 0,5 \\
\hline $\mathbf{C}$ & 4,811 & 0,924 & 15,703 & 0,12 & $-0,14$ & 0,22 & 8,8 & 6,8 & 7,3 & 1,8 & 2,6 & $-1,8$ \\
\hline $\mathbf{C}$ & 2,492 & $-0,064$ & 15,054 & 0,15 & 0,00 & 0,09 & 11,6 & 7,3 & 10,1 & 0,2 & 4,5 & $-1,4$ \\
\hline $\mathbf{H}$ & 1,789 & $-1,735$ & 16,028 & 0,03 & 0,06 & $-0,03$ & 1,4 & 3,5 & 1,9 & 1,0 & $-0,6$ & $-1,4$ \\
\hline $\mathbf{C}$ & 1,071 & 1,026 & 13,207 & $-0,01$ & $-0,19$ & 0,16 & 7,1 & 8,3 & 10,2 & $-0,9$ & 2,6 & $-3,9$ \\
\hline $\mathbf{H}$ & $-0,771$ & 0,257 & 12,708 & 0,03 & 0,03 & 0,00 & 3,1 & 1,5 & 1,0 & 1,1 & 0,4 & 0,2 \\
\hline $\mathbf{H}$ & 7,670 & 0,718 & 17,910 & 0,11 & 0,06 & 0,04 & 1,5 & 0,5 & 0,6 & 0,7 & 0,8 & 0,5 \\
\hline
\end{tabular}


Table S15: atomic coordinates, dipole moments and polarizabilities of the central 1,4nitrophenol within a three-molecule cluster using ADIM, all values in a.u.

\begin{tabular}{ccccccccccccc}
\hline Atom & $\mathbf{x}$ & $\mathbf{y}$ & $\mathbf{z}$ & $\boldsymbol{\mu}_{\mathbf{x}}$ & $\boldsymbol{\mu}_{\mathbf{y}}$ & $\boldsymbol{\mu}_{\mathrm{z}}$ & $\boldsymbol{\alpha}_{\mathbf{x x}}$ & $\boldsymbol{\alpha}_{\mathrm{yy}}$ & $\boldsymbol{\alpha}_{\mathbf{z z}}$ & $\boldsymbol{\alpha}_{\mathbf{x y}}$ & $\boldsymbol{\alpha}_{\mathbf{x z}}$ & $\boldsymbol{\alpha}_{\mathbf{y z}}$ \\
\hline $\mathbf{N}$ & 0,481 & 4,298 & 10,038 & 0,55 & $-0,47$ & 0,74 & 11,2 & 7,3 & 12,3 & $-2,6$ & 7,3 & $-5,2$ \\
\hline $\mathbf{0}$ & 6,088 & $-0,236$ & 17,582 & 0,23 & 0,68 & $-0,40$ & 8,0 & 5,8 & 10,8 & $-1,7$ & 5,2 & $-1,0$ \\
\hline $\mathbf{0}$ & 1,318 & 6,173 & 8,948 & $-0,35$ & $-0,80$ & 0,48 & 5,2 & 8,4 & 7,8 & $-0,5$ & 0,0 & $-3,3$ \\
\hline $\mathbf{0}$ & $-1,582$ & 3,420 & 9,533 & 0,86 & 0,37 & 0,21 & 10,2 & 4,3 & 6,0 & 1,2 & 3,4 & 0,1 \\
\hline $\mathbf{C}$ & 2,015 & 3,129 & 11,978 & 0,22 & $-0,17$ & 0,29 & 10,2 & 7,9 & 9,2 & 0,4 & 3,3 & $-2,6$ \\
\hline $\mathbf{C}$ & 4,350 & 4,100 & 12,554 & 0,19 & 0,02 & 0,11 & 10,8 & 5,7 & 9,2 & $-0,3$ & 4,5 & $-1,1$ \\
\hline $\mathbf{H}$ & 5,082 & 5,723 & 11,523 & $-0,03$ & $-0,03$ & 0,01 & 1,5 & 2,4 & 1,5 & 0,9 & $-0,5$ & $-0,8$ \\
\hline $\mathbf{C}$ & 5,744 & 3,010 & 14,431 & $-0,02$ & $-0,12$ & 0,13 & 8,5 & 8,0 & 11,2 & $-0,5$ & 3,0 & $-2,1$ \\
\hline $\mathbf{H}$ & 7,587 & 3,782 & 14,926 & $-0,09$ & $-0,03$ & $-0,02$ & 4,2 & 1,5 & 1,8 & 1,3 & 1,6 & 0,5 \\
\hline $\mathbf{C}$ & 4,811 & 0,924 & 15,703 & 0,13 & $-0,12$ & 0,21 & 8,8 & 7,0 & 7,1 & 2,2 & 2,6 & $-1,4$ \\
\hline $\mathbf{C}$ & 2,492 & $-0,064$ & 15,054 & 0,13 & 0,01 & 0,08 & 10,7 & 7,2 & 9,4 & 0,1 & 3,9 & $-1,2$ \\
\hline $\mathbf{H}$ & 1,789 & $-1,735$ & 16,028 & 0,03 & 0,06 & $-0,03$ & 1,5 & 3,5 & 1,9 & 1,1 & $-0,6$ & $-1,4$ \\
\hline $\mathbf{C}$ & 1,071 & 1,026 & 13,207 & $-0,01$ & $-0,17$ & 0,15 & 7,1 & 8,3 & 9,6 & $-0,7$ & 2,1 & $-3,3$ \\
\hline $\mathbf{H}$ & $-0,771$ & 0,257 & 12,708 & 0,03 & 0,03 & 0,00 & 3,0 & 1,5 & 1,0 & 1,1 & 0,5 & 0,2 \\
\hline $\mathbf{H}$ & 7,670 & 0,718 & 17,910 & 0,09 & 0,04 & 0,03 & 1,6 & 0,6 & 0,8 & 0,5 & 1,2 & 0,6 \\
\hline
\end{tabular}


Table S16: atomic coordinates, dipole moments and polarizabilities of the isolated amino acids within the tripeptide, all values in a.u.

\begin{tabular}{|c|c|c|c|c|c|c|c|c|c|c|c|c|}
\hline Atom & $\mathbf{x}$ & $\mathrm{y}$ & $\mathbf{z}$ & $\mu_{\mathrm{x}}$ & $\mu_{\mathrm{y}}$ & $\mu_{\mathrm{z}}$ & $\alpha_{x x}$ & $\alpha_{y y}$ & $\alpha_{\mathrm{zz}}$ & $\alpha_{\mathrm{xy}}$ & $\alpha_{x z}$ & $\alpha_{\mathrm{yz}}$ \\
\hline \multicolumn{13}{|c|}{ Ending Chain Glycyl } \\
\hline $\mathrm{N}$ & 1,848 & 11,737 & 29,575 & 0,00 & 0,00 & 0,29 & 7,4 & 11,4 & 12,6 & $-1,0$ & 1,7 & $-1,3$ \\
\hline $\mathrm{H}$ & $-0,052$ & 11,905 & 29,774 & $-0,04$ & $-0,01$ & 0,02 & 1,8 & 0,5 & 0,4 & $-0,3$ & 0,1 & 0,0 \\
\hline C & 3,116 & 10,007 & 31,286 & 0,06 & 0,06 & $-0,13$ & 5,7 & 4,6 & 6,2 & 0,3 & $-1,3$ & $-0,9$ \\
\hline $\mathrm{H}$ & 4,787 & 10,918 & 32,070 & $-0,10$ & $-0,03$ & $-0,05$ & 3,8 & 1,6 & 1,7 & 1,0 & 0,8 & 0,3 \\
\hline $\mathrm{H}$ & 3,727 & 8,339 & 30,247 & $-0,05$ & 0,11 & 0,02 & 1,9 & 3,4 & 1,9 & $-1,2$ & $-0,7$ & 1,0 \\
\hline $\mathrm{C}$ & 1,358 & 9,218 & 33,429 & 0,30 & $-0,17$ & 0,13 & 5,2 & 3,0 & 5,7 & 0,2 & $-1,7$ & $-1,8$ \\
\hline 0 & 2,320 & 7,882 & 35,139 & $-0,72$ & 0,17 & 0,07 & 5,4 & 7,0 & 9,0 & $-0,8$ & 1,1 & $-2,8$ \\
\hline 0 & $-0,871$ & 9,880 & 33,333 & 1,39 & $-0,39$ & 0,03 & 11,3 & 6,1 & 5,7 & $-1,1$ & $-0,4$ & 0,3 \\
\hline $\mathrm{H}$ & 1,235 & 7,317 & 36,564 & $-0,04$ & $-0,03$ & 0,07 & 0,5 & 0,5 & 1,2 & 0,3 & $-0,6$ & $-0,4$ \\
\hline $\mathrm{H}$ & 2,719 & 13,104 & 28,545 & 0,00 & 0,01 & $-0,02$ & 0,8 & 1,7 & 1,2 & 0,6 & $-0,5$ & $-0,9$ \\
\hline \multicolumn{13}{|c|}{ Middle Chain Glycyl } \\
\hline $\mathbf{N}$ & $-0,493$ & 14,367 & 25,291 & 0,14 & 0,14 & 0,10 & 11,3 & 11,1 & 10,6 & 0,7 & 3,6 & 0,2 \\
\hline $\mathrm{H}$ & $-2,312$ & 14,170 & 25,865 & $-0,02$ & 0,00 & 0,01 & 2,4 & 0,5 & 0,6 & 0,3 & $-0,3$ & $-0,1$ \\
\hline 0 & 5,344 & 13,841 & 28,366 & $-1,28$ & $-0,14$ & $-0,02$ & 12,7 & 5,3 & 5,8 & 0,1 & 0,8 & 0,4 \\
\hline C & 1,345 & 15,482 & 26,998 & $-0,08$ & 0,10 & $-0,08$ & 5,8 & 5,3 & 5,1 & $-1,2$ & 1,2 & $-0,7$ \\
\hline $\mathrm{H}$ & 0,339 & 16,589 & 28,412 & 0,05 & $-0,06$ & $-0,09$ & 2,1 & 2,4 & 2,7 & $-0,9$ & $-0,9$ & 1,2 \\
\hline $\mathrm{H}$ & 2,534 & 16,775 & 25,926 & $-0,06$ & $-0,08$ & 0,04 & 2,0 & 2,8 & 1,9 & 1,1 & $-0,6$ & $-0,9$ \\
\hline $\mathrm{C}$ & 3,031 & 13,597 & 28,345 & $-0,11$ & 0,02 & $-0,07$ & 7,0 & 5,2 & 4,6 & $-1,3$ & 1,5 & $-1,7$ \\
\hline $\mathrm{H}$ & 0,045 & 13,871 & 23,513 & 0,01 & 0,00 & $-0,02$ & 0,5 & 0,6 & 2,2 & $-0,1$ & $-0,2$ & 0,5 \\
\hline $\mathrm{H}$ & 2,090 & 11,946 & 29,133 & 0,06 & 0,08 & $-0,04$ & 1,9 & 3,5 & 1,9 & 1,0 & $-0,6$ & $-1,2$ \\
\hline \multicolumn{13}{|c|}{ Ending Chain Leucyl } \\
\hline $\mathrm{N}$ & $-1,777$ & 14,324 & 18,749 & 0,55 & 0,17 & 0,55 & 6,9 & 9,7 & 12,8 & $-0,2$ & 1,3 & $-3,6$ \\
\hline 0 & 2,281 & 14,234 & 22,065 & $-1,21$ & $-0,19$ & 0,43 & 11,3 & 6,1 & 4,7 & 1,9 & $-0,8$ & $-0,5$ \\
\hline $\mathrm{C}$ & $-1,947$ & 12,872 & 21,167 & $-0,09$ & $-0,15$ & $-0,14$ & 5,2 & 9,9 & 5,6 & 1,1 & 1,5 & 0,3 \\
\hline $\mathrm{H}$ & $-3,787$ & 13,162 & 22,040 & 0,10 & $-0,04$ & $-0,06$ & 3,3 & 1,6 & 1,5 & $-0,5$ & $-1,0$ & 0,2 \\
\hline $\mathrm{C}$ & 0,140 & 13,889 & 22,879 & $-0,12$ & $-0,03$ & $-0,06$ & 6,6 & 4,2 & 5,7 & 1,8 & 1,4 & 1,4 \\
\hline C & $-1,555$ & 10,063 & 20,568 & 0,01 & $-0,10$ & $-0,01$ & 4,1 & 11,0 & 6,2 & 0,9 & $-0,5$ & $-2,1$ \\
\hline $\mathrm{H}$ & $-2,883$ & 9,518 & 19,094 & 0,08 & 0,05 & 0,10 & 2,1 & 1,7 & 3,1 & 0,6 & 1,3 & 0,7 \\
\hline $\mathrm{H}$ & 0,344 & 9,816 & 19,813 & $-0,12$ & 0,02 & 0,07 & 3,2 & 1,5 & 1,9 & $-0,4$ & $-1,3$ & 0,1 \\
\hline C & $-1,890$ & 8,262 & 22,815 & $-0,02$ & $-0,05$ & $-0,01$ & 7,1 & 9,6 & 6,4 & $-0,2$ & $-2,2$ & $-1,5$ \\
\hline $\mathrm{H}$ & $-0,586$ & 8,831 & 24,302 & $-0,10$ & $-0,05$ & $-0,12$ & 2,6 & 1,7 & 3,0 & 0,5 & 1,5 & 0,5 \\
\hline C & $-1,222$ & 5,573 & 21,998 & $-0,01$ & 0,04 & 0,00 & 5,0 & 9,4 & 4,9 & $-1,2$ & $-0,3$ & 0,7 \\
\hline $\mathrm{H}$ & $-1,425$ & 4,299 & 23,602 & 0,01 & 0,11 & $-0,11$ & 1,3 & 3,8 & 2,9 & 0,1 & $-0,1$ & $-1,9$ \\
\hline $\mathrm{H}$ & 0,722 & 5,525 & 21,324 & $-0,14$ & 0,02 & 0,05 & 4,2 & 1,7 & 1,7 & $-0,8$ & $-1,1$ & 0,3 \\
\hline $\mathrm{H}$ & $-2,486$ & 4,982 & 20,486 & 0,09 & 0,06 & 0,12 & 2,2 & 2,3 & 3,0 & 0,8 & 1,2 & 1,2 \\
\hline $\mathrm{C}$ & $-4,559$ & 8,363 & 23,867 & 0,05 & $-0,02$ & $-0,03$ & 8,1 & 5,0 & 5,5 & 0,3 & $-1,9$ & $-0,3$ \\
\hline $\mathrm{H}$ & $-5,905$ & 7,968 & 22,361 & 0,12 & 0,03 & 0,10 & 3,5 & 1,5 & 2,3 & 0,6 & 1,4 & 0,3 \\
\hline $\mathrm{H}$ & $-4,922$ & 10,236 & 24,639 & 0,05 & $-0,14$ & $-0,07$ & 1,9 & 3,6 & 2,0 & $-0,8$ & $-0,7$ & 1,2 \\
\hline $\mathrm{H}$ & $-4,764$ & 6,958 & 25,356 & 0,03 & 0,10 & $-0,11$ & 1,7 & 2,8 & 3,4 & 0,7 & $-0,9$ & $-1,8$ \\
\hline $\mathrm{H}$ & $-0,040$ & 14,103 & 17,965 & 0,00 & $-0,01$ & 0,00 & 1,6 & 0,5 & 1,0 & 0,1 & $-0,8$ & $-0,1$ \\
\hline $\mathrm{H}$ & $-2,096$ & 16,184 & 19,092 & $-0,01$ & $-0,02$ & $-0,01$ & 0,6 & 3,1 & 0,6 & $-0,2$ & 0,0 & 0,1 \\
\hline $\mathrm{H}$ & $-0,420$ & 14,394 & 24,793 & 0,04 & $-0,02$ & $-0,10$ & 1,4 & 1,7 & 4,3 & $-0,1$ & $-0,6$ & 1,0 \\
\hline
\end{tabular}


Table S17: atomic coordinates, dipole moments and polarizabilities of the tripeptide calculated with QTAIM, all values in a.u.

\begin{tabular}{|c|c|c|c|c|c|c|c|c|c|c|c|c|}
\hline Atom & $x$ & $y$ & $\mathbf{z}$ & $\mu_{x}$ & $\mu_{y}$ & $\mu_{\mathrm{z}}$ & $\alpha_{x x}$ & $\alpha_{y y}$ & $\alpha_{\mathrm{zz}}$ & $\alpha_{x y}$ & $\alpha_{x z}$ & $\alpha_{y z}$ \\
\hline $\mathrm{N}$ & $-1,777$ & 14,324 & 18,749 & 0,57 & 0,17 & 0,55 & 6,9 & 9,3 & 13,8 & $-0,2$ & 1,7 & $-3,6$ \\
\hline $\mathrm{N}$ & $-0,493$ & 14,367 & 25,291 & 0,18 & 0,19 & 0,23 & 7,8 & 6,8 & 18,9 & 0,8 & 2,9 & 3,4 \\
\hline $\mathrm{H}$ & $-2,312$ & 14,170 & 25,865 & $-0,03$ & 0,00 & 0,01 & 1,7 & 0,4 & 0,7 & 0,1 & $-0,4$ & 0,0 \\
\hline $\mathrm{N}$ & 1,848 & 11,737 & 29,575 & 0,01 & $-0,13$ & 0,39 & 6,3 & 12,5 & 12,9 & 1,4 & 0,3 & $-7,0$ \\
\hline $\mathrm{H}$ & $-0,052$ & 11,905 & 29,774 & $-0,04$ & 0,00 & 0,02 & 1,5 & 0,3 & 0,4 & $-0,1$ & 0,0 & 0,0 \\
\hline 0 & 2,281 & 14,234 & 22,065 & $-1,37$ & $-0,22$ & 0,54 & 9,4 & 5,6 & 6,6 & 1,2 & $-2,2$ & $-0,7$ \\
\hline 0 & 5,344 & 13,841 & 28,366 & $-1,46$ & $-0,17$ & $-0,04$ & 12,4 & 5,9 & 5,7 & 2,0 & 0,3 & 0,2 \\
\hline C & $-1,947$ & 12,872 & 21,167 & $-0,08$ & $-0,14$ & $-0,14$ & 3,9 & 9,2 & 6,3 & 1,0 & 3,0 & 1,1 \\
\hline $\mathrm{H}$ & $-3,787$ & 13,162 & 22,040 & 0,11 & $-0,03$ & $-0,06$ & 3,2 & 1,5 & 1,8 & $-0,5$ & $-0,6$ & 0,2 \\
\hline C & 0,140 & 13,889 & 22,879 & $-0,24$ & $-0,01$ & 0,21 & 4,9 & 2,9 & 8,7 & 1,5 & 2,0 & 1,9 \\
\hline C & 1,345 & 15,482 & 26,998 & 0,02 & 0,11 & $-0,07$ & 4,4 & 5,7 & 6 & $-1,1$ & 2,2 & $-1,1$ \\
\hline $\mathrm{H}$ & 0,339 & 16,589 & 28,412 & 0,06 & $-0,05$ & $-0,07$ & 1,7 & 2,6 & 2,7 & $-0,9$ & $-0,7$ & 1,3 \\
\hline $\mathrm{H}$ & 2,534 & 16,775 & 25,926 & $-0,02$ & $-0,06$ & 0,00 & 1,4 & 2,6 & 1,3 & 1,0 & $-0,2$ & $-0,7$ \\
\hline C & 3,031 & 13,597 & 28,345 & $-0,29$ & $-0,20$ & 0,09 & 5,4 & 5,2 & 5,2 & $-0,2$ & 1,3 & $-3,3$ \\
\hline C & 3,116 & 10,007 & 31,286 & 0,13 & 0,03 & $-0,09$ & 4,7 & 4,7 & 7,0 & 0,4 & $-1,3$ & $-1,5$ \\
\hline $\mathrm{H}$ & 4,787 & 10,918 & 32,070 & $-0,04$ & $-0,01$ & $-0,04$ & 2,6 & 1,2 & 1,7 & 0,5 & 1,0 & 0,2 \\
\hline $\mathrm{H}$ & 3,727 & 8,339 & 30,247 & $-0,03$ & 0,08 & 0,02 & 1,5 & 3,2 & 1,8 & $-0,9$ & $-0,4$ & 0,7 \\
\hline C & 1,358 & 9,218 & 33,429 & 0,30 & $-0,18$ & 0,15 & 4,7 & 3,1 & 6,5 & 0,3 & $-1,7$ & $-2,2$ \\
\hline 0 & 2,320 & 7,882 & 35,139 & $-0,71$ & 0,14 & 0,10 & 5,2 & 7,0 & 9,8 & $-0,9$ & 1,3 & $-3,3$ \\
\hline 0 & $-0,871$ & 9,880 & 33,333 & 1,39 & $-0,41$ & 0,05 & 10,5 & 5,9 & 6,4 & $-0,9$ & $-0,5$ & 0,0 \\
\hline C & $-1,555$ & 10,063 & 20,568 & 0,02 & $-0,09$ & $-0,02$ & 4,2 & 12,8 & 5,9 & $-1,0$ & $-0,6$ & 0,2 \\
\hline $\mathrm{H}$ & $-2,883$ & 9,518 & 19,094 & 0,08 & 0,04 & 0,10 & 2,1 & 1,7 & 3,4 & 0,6 & 1,5 & 0,8 \\
\hline $\mathrm{H}$ & 0,344 & 9,816 & 19,813 & $-0,11$ & 0,02 & 0,07 & 2,9 & 1,4 & 2,0 & $-0,4$ & $-1,2$ & 0,2 \\
\hline C & $-1,890$ & 8,262 & 22,815 & 0,01 & $-0,06$ & $-0,01$ & 4,2 & 10,1 & 7,0 & 1,5 & $-1,2$ & $-0,6$ \\
\hline $\mathrm{H}$ & $-0,586$ & 8,831 & 24,302 & $-0,09$ & $-0,04$ & $-0,11$ & 2,4 & 1,6 & 2,9 & 0,6 & 1,5 & 0,6 \\
\hline C & $-1,222$ & 5,573 & 21,998 & $-0,01$ & 0,04 & 0,00 & 4,8 & 9,5 & 4,9 & $-1,0$ & $-0,2$ & 1,0 \\
\hline $\mathrm{H}$ & $-1,425$ & 4,299 & 23,602 & 0,01 & 0,11 & $-0,11$ & 1,3 & 3,8 & 2,8 & 0,1 & $-0,1$ & $-1,8$ \\
\hline $\mathrm{H}$ & 0,722 & 5,525 & 21,324 & $-0,14$ & 0,03 & 0,05 & 4,0 & 1,7 & 1,7 & $-0,8$ & $-1,0$ & 0,4 \\
\hline $\mathrm{H}$ & $-2,486$ & 4,982 & 20,486 & 0,09 & 0,06 & 0,12 & 2,2 & 2,4 & 3,1 & 0,9 & 1,3 & 1,3 \\
\hline C & $-4,559$ & 8,363 & 23,867 & 0,06 & $-0,02$ & $-0,04$ & 8,1 & 4,6 & 4,7 & 0,9 & $-1,1$ & $-0,8$ \\
\hline $\mathrm{H}$ & $-5,905$ & 7,968 & 22,361 & 0,12 & 0,03 & 0,10 & 3,6 & 1,5 & 2,4 & 0,7 & 1,5 & 0,4 \\
\hline $\mathrm{H}$ & $-4,922$ & 10,236 & 24,639 & 0,05 & $-0,15$ & $-0,08$ & 2,0 & 2,6 & 1,9 & $-0,8$ & $-0,4$ & 0,9 \\
\hline $\mathrm{H}$ & $-4,764$ & 6,958 & 25,356 & 0,04 & 0,10 & $-0,11$ & 1,7 & 2,8 & 3,2 & 0,8 & $-0,8$ & $-1,7$ \\
\hline $\mathrm{H}$ & $-0,040$ & 14,103 & 17,965 & 0,01 & $-0,01$ & 0,00 & 1,5 & 0,5 & 1,1 & 0,1 & $-0,8$ & $-0,1$ \\
\hline $\mathrm{H}$ & $-2,096$ & 16,184 & 19,092 & $-0,01$ & $-0,02$ & $-0,01$ & 0,6 & 3,0 & 0,6 & $-0,2$ & 0,0 & 0,0 \\
\hline $\mathrm{H}$ & 1,235 & 7,317 & 36,564 & $-0,05$ & $-0,03$ & 0,08 & 0,5 & 0,4 & 1,3 & 0,3 & $-0,6$ & $-0,5$ \\
\hline
\end{tabular}


Table S18: atomic coordinates, dipole moments and polarizabilities of the tripeptide calculated with ADIM, all values in a.u.

\begin{tabular}{|c|c|c|c|c|c|c|c|c|c|c|c|c|}
\hline Atom & $x$ & $y$ & $\mathbf{z}$ & $\mu_{x}$ & $\mu_{y}$ & $\mu_{\mathrm{z}}$ & $\alpha_{x x}$ & $\alpha_{y y}$ & $\alpha_{\mathrm{zz}}$ & $\alpha_{x y}$ & $\alpha_{x z}$ & $\alpha_{\mathrm{yz}}$ \\
\hline $\mathrm{N}$ & 1,848 & 11,737 & 29,575 & $-0,01$ & $-0,26$ & 0,55 & 4,7 & 14,1 & 13,7 & 3,9 & 0,5 & $-6,4$ \\
\hline $\mathrm{H}$ & $-0,052$ & 11,905 & 29,774 & $-0,05$ & $-0,01$ & 0,02 & 1,6 & 0,4 & 0,4 & $-0,2$ & 0,1 & $-0,1$ \\
\hline $\mathrm{C}$ & 3,116 & 10,007 & 31,286 & 0,08 & 0,02 & $-0,08$ & 4,7 & 5,0 & 6,9 & 0,2 & $-1,3$ & $-2,0$ \\
\hline $\mathrm{H}$ & 4,787 & 10,918 & 32,070 & $-0,05$ & $-0,02$ & $-0,03$ & 3,3 & 1,5 & 2,0 & 0,8 & 1,1 & $-0,1$ \\
\hline $\mathrm{H}$ & 3,727 & 8,339 & 30,247 & $-0,04$ & 0,09 & 0,02 & 1,7 & 3,6 & 1,8 & $-1,1$ & $-0,4$ & 0,9 \\
\hline C & 1,358 & 9,218 & 33,429 & 0,30 & $-0,18$ & 0,15 & 4,7 & 3,0 & 6,3 & 0,2 & $-1,9$ & $-2,2$ \\
\hline 0 & 2,320 & 7,882 & 35,139 & $-0,71$ & 0,15 & 0,09 & 5,1 & 7,0 & 9,7 & $-0,9$ & 1,3 & $-3,2$ \\
\hline 0 & $-0,871$ & 9,880 & 33,333 & 1,39 & $-0,40$ & 0,05 & 10,4 & 5,7 & 6,3 & $-0,9$ & $-0,7$ & 0,0 \\
\hline $\mathrm{H}$ & 1,235 & 7,317 & 36,564 & $-0,04$ & $-0,03$ & 0,08 & 0,5 & 0,5 & 1,3 & 0,3 & $-0,6$ & $-0,5$ \\
\hline $\mathrm{N}$ & $-0,493$ & 14,367 & 25,291 & 0,19 & 0,18 & 0,39 & 5,2 & 7,9 & 19,1 & $-0,7$ & 4,3 & 2,3 \\
\hline $\mathrm{H}$ & $-2,312$ & 14,170 & 25,865 & $-0,05$ & 0,00 & 0,02 & 2,0 & 0,4 & 0,7 & 0,2 & $-0,5$ & 0,0 \\
\hline 0 & 5,344 & 13,841 & 28,366 & $-1,25$ & $-0,12$ & $-0,06$ & 13,1 & 5,0 & 5,3 & 1,2 & $-0,5$ & 0,0 \\
\hline C & 1,345 & 15,482 & 26,998 & $-0,04$ & 0,09 & $-0,04$ & 4,4 & 6,0 & 6,6 & $-1,0$ & 2,2 & $-1,0$ \\
\hline $\mathrm{H}$ & 0,339 & 16,589 & 28,412 & 0,05 & $-0,05$ & $-0,08$ & 1,7 & 3,0 & 2,7 & $-0,8$ & $-0,8$ & 1,6 \\
\hline $\mathrm{H}$ & 2,534 & 16,775 & 25,926 & $-0,03$ & $-0,06$ & 0,03 & 1,7 & 3,2 & 1,9 & 1,1 & $-0,4$ & $-0,9$ \\
\hline C & 3,031 & 13,597 & 28,345 & $-0,18$ & $-0,02$ & $-0,10$ & 3,2 & 7,8 & 5,2 & 1,7 & $-1,4$ & $-4,9$ \\
\hline $\mathrm{N}$ & $-1,777$ & 14,324 & 18,749 & 0,55 & 0,16 & 0,55 & 6,7 & 9,1 & 14,5 & $-0,5$ & 1,8 & $-3,5$ \\
\hline 0 & 2,281 & 14,234 & 22,065 & $-1,20$ & $-0,19$ & 0,40 & 9,6 & 4,6 & 6,1 & 1,6 & $-3,3$ & $-0,4$ \\
\hline C & $-1,947$ & 12,872 & 21,167 & $-0,10$ & $-0,15$ & $-0,13$ & 5,2 & 8,8 & 7,3 & 1,8 & 2,7 & 1,0 \\
\hline $\mathrm{H}$ & $-3,787$ & 13,162 & 22,040 & 0,10 & $-0,04$ & $-0,06$ & 3,3 & 1,3 & 1,5 & $-0,4$ & $-0,3$ & 0,2 \\
\hline C & 0,140 & 13,889 & 22,879 & $-0,23$ & $-0,09$ & $-0,08$ & 1,5 & 1,5 & 12,3 & 0,5 & 0,2 & 2,3 \\
\hline C & $-1,555$ & 10,063 & 20,568 & 0,01 & $-0,10$ & $-0,01$ & 3,9 & 10,7 & 6,5 & 1,3 & $-0,3$ & $-1,5$ \\
\hline $\mathrm{H}$ & $-2,883$ & 9,518 & 19,094 & 0,08 & 0,04 & 0,10 & 2,1 & 1,7 & 3,4 & 0,6 & 1,5 & 0,8 \\
\hline $\mathrm{H}$ & 0,344 & 9,816 & 19,813 & $-0,12$ & 0,02 & 0,07 & 2,9 & 1,5 & 2,1 & $-0,3$ & $-1,4$ & 0,3 \\
\hline C & $-1,890$ & 8,262 & 22,815 & $-0,03$ & $-0,04$ & $-0,01$ & 6,5 & 9,8 & 6,3 & 0,5 & $-1,9$ & $-1,2$ \\
\hline $\mathrm{H}$ & $-0,586$ & 8,831 & 24,302 & $-0,11$ & $-0,05$ & $-0,13$ & 2,4 & 1,9 & 3,3 & 0,6 & 1,8 & 0,9 \\
\hline C & $-1,222$ & 5,573 & 21,998 & $-0,01$ & 0,03 & 0,00 & 4,8 & 9,6 & 5,0 & $-0,8$ & $-0,3$ & 1,0 \\
\hline $\mathrm{H}$ & $-1,425$ & 4,299 & 23,602 & 0,01 & 0,11 & $-0,11$ & 1,3 & 3,8 & 2,8 & 0,2 & 0,0 & $-1,8$ \\
\hline $\mathrm{H}$ & 0,722 & 5,525 & 21,324 & $-0,14$ & 0,02 & 0,05 & 4,0 & 1,8 & 1,7 & $-0,8$ & $-1,1$ & 0,4 \\
\hline $\mathrm{H}$ & $-2,486$ & 4,982 & 20,486 & 0,09 & 0,06 & 0,12 & 2,2 & 2,4 & 3,1 & 0,9 & 1,3 & 1,3 \\
\hline C & $-4,559$ & 8,363 & 23,867 & 0,04 & $-0,01$ & $-0,03$ & 8,1 & 5,0 & 5,3 & 0,7 & $-1,3$ & $-0,2$ \\
\hline $\mathrm{H}$ & $-5,905$ & 7,968 & 22,361 & 0,12 & 0,03 & 0,10 & 3,7 & 1,5 & 2,4 & 0,7 & 1,6 & 0,4 \\
\hline $\mathrm{H}$ & $-4,922$ & 10,236 & 24,639 & 0,05 & $-0,14$ & $-0,07$ & 1,8 & 3,5 & 1,9 & $-0,3$ & $-0,5$ & 1,1 \\
\hline $\mathrm{H}$ & $-4,764$ & 6,958 & 25,356 & 0,03 & 0,10 & $-0,11$ & 1,7 & 2,8 & 3,2 & 0,9 & $-0,7$ & $-1,8$ \\
\hline $\mathrm{H}$ & $-0,040$ & 14,103 & 17,965 & 0,00 & $-0,01$ & 0,00 & 1,5 & 0,5 & 1,1 & 0,1 & $-0,9$ & $-0,1$ \\
\hline $\mathrm{H}$ & $-2,096$ & 16,184 & 19,092 & $-0,01$ & $-0,02$ & $-0,01$ & 0,5 & 2,9 & 0,6 & $-0,3$ & 0,0 & 0,0 \\
\hline
\end{tabular}


Table S19: atomic coordinates, dipole moments and polarizabilities of the isolated aromatic compounds within the polyaromatic molecule, all values in a.u.

\begin{tabular}{|c|c|c|c|c|c|c|c|c|c|c|c|c|}
\hline Atom & $\mathbf{x}$ & $\mathrm{y}$ & $\mathbf{z}$ & $\mu_{\mathrm{x}}$ & $\mu_{\mathrm{y}}$ & $\mu_{\mathrm{z}}$ & $\alpha_{x x}$ & $\alpha_{\mathrm{yy}}$ & $\alpha_{\mathrm{zz}}$ & $\alpha_{x y}$ & $\alpha_{x z}$ & $\alpha_{\mathrm{yz}}$ \\
\hline \multicolumn{13}{|c|}{ Ending Chain Toluene } \\
\hline C & 15,490 & 7,805 & 1,806 & $-0,01$ & $-0,01$ & 0,00 & 9,6 & 4,2 & 4,6 & $-0,4$ & 0,8 & 0,1 \\
\hline $\mathrm{C}$ & 12,811 & 7,925 & 1,390 & 0,11 & 0,00 & 0,02 & 11,9 & 9,3 & 5,5 & 0,0 & 0,9 & $-2,2$ \\
\hline C & 11,262 & 5,916 & 2,139 & 0,00 & 0,07 & $-0,04$ & 11,7 & 8,5 & 6,4 & 1,4 & 0,1 & $-1,0$ \\
\hline C & 8,681 & 6,053 & 1,753 & 0,05 & 0,05 & $-0,01$ & 10,9 & 8,8 & 6,8 & $-0,8$ & 1,0 & $-1,4$ \\
\hline C & 7,620 & 8,155 & 0,625 & 0,08 & $-0,01$ & 0,01 & 9,6 & 10,2 & 7,1 & 0,3 & 0,3 & $-1,8$ \\
\hline $\mathrm{C}$ & 9,142 & 10,158 & $-0,111$ & 0,04 & $-0,05$ & 0,03 & 11,2 & 8,8 & 6,6 & 0,9 & 0,3 & $-1,2$ \\
\hline C & 11,723 & 10,048 & 0,268 & $-0,02$ & $-0,08$ & 0,03 & 11,2 & 8,8 & 6,9 & $-1,4$ & 1,3 & $-1,4$ \\
\hline H & 12,108 & 4,258 & 3,016 & $-0,05$ & 0,09 & $-0,05$ & 1,7 & 3,9 & 2,0 & $-1,2$ & 0,6 & $-1,4$ \\
\hline $\mathrm{H}$ & 7,526 & 4,466 & 2,370 & 0,07 & 0,08 & $-0,03$ & 2,6 & 3,6 & 1,6 & 1,9 & $-0,7$ & $-0,9$ \\
\hline $\mathrm{H}$ & 5,587 & 8,205 & 0,307 & 0,11 & $-0,01$ & 0,02 & 5,4 & 1,2 & 1,4 & $-0,2$ & 0,7 & 0,0 \\
\hline $\mathrm{H}$ & 8,322 & 11,823 & $-0,999$ & 0,05 & $-0,09$ & 0,05 & 1,9 & 3,9 & 2,0 & $-1,4$ & 0,8 & $-1,4$ \\
\hline $\mathrm{H}$ & 12,973 & 11,585 & $-0,289$ & $-0,06$ & $-0,09$ & 0,03 & 2,3 & 3,6 & 1,6 & 1,7 & $-0,7$ & $-0,9$ \\
\hline $\mathrm{H}$ & 16,356 & 9,498 & 1,117 & $-0,09$ & $-0,12$ & 0,04 & 2,8 & 2,8 & 1,5 & 1,6 & $-0,5$ & $-0,5$ \\
\hline $\mathrm{H}$ & 16,254 & 6,210 & 0,825 & $-0,08$ & 0,11 & 0,06 & 2,9 & 2,9 & 1,8 & $-1,6$ & $-0,8$ & 1,0 \\
\hline $\mathrm{H}$ & 15,858 & 7,619 & 3,786 & $-0,05$ & 0,02 & $-0,14$ & 2,3 & 1,2 & 4,1 & $-0,2$ & 1,5 & $-0,2$ \\
\hline \multicolumn{13}{|c|}{ Middle Chain Anthracene } \\
\hline C & 21,680 & 0,814 & 3,011 & 0,05 & 0,05 & 0,02 & 9,2 & 13,9 & 6,2 & $-1,8$ & 0,8 & $-0,4$ \\
\hline C & 20,415 & 3,023 & 2,695 & 0,05 & 0,03 & 0,01 & 8,6 & 16,9 & 5,8 & $-0,5$ & 0,7 & $-0,1$ \\
\hline C & 21,661 & 5,391 & 3,031 & 0,01 & 0,00 & 0,00 & 11,6 & 17,5 & 4,5 & $-0,7$ & 1,8 & 0,0 \\
\hline $\mathrm{C}$ & 20,367 & 7,712 & 2,741 & $-0,13$ & 0,00 & $-0,02$ & 11,3 & 19,1 & 4,2 & 1,3 & 1,7 & 0,3 \\
\hline $\mathrm{C}$ & 21,660 & 10,037 & 3,035 & 0,01 & 0,00 & 0,00 & 11,6 & 19,5 & 4,4 & 1,6 & 1,8 & 0,3 \\
\hline C & 20,414 & 12,403 & 2,732 & 0,06 & $-0,03$ & 0,02 & 7,8 & 17,4 & 5,8 & $-0,3$ & 0,5 & $-0,1$ \\
\hline C & 21,673 & 14,618 & 3,033 & 0,05 & $-0,05$ & 0,01 & 9,2 & 13,5 & 6,2 & 1,3 & 0,8 & 0,3 \\
\hline $\mathrm{C}$ & 17,718 & 7,733 & 2,195 & 0,03 & 0,01 & 0,01 & 9,0 & 4,8 & 4,5 & 0,4 & 1,0 & 0,1 \\
\hline C & 24,267 & 0,814 & 3,685 & $-0,05$ & 0,05 & $-0,02$ & 9,1 & 13,4 & 6,2 & 1,4 & 0,8 & 0,3 \\
\hline C & 25,533 & 3,023 & 4,001 & $-0,06$ & 0,03 & $-0,01$ & 7,8 & 17,4 & 5,8 & $-0,3$ & 0,5 & $-0,1$ \\
\hline C & 24,287 & 5,391 & 3,665 & $-0,01$ & 0,00 & 0,00 & 11,5 & 19,6 & 4,4 & 1,5 & 1,8 & 0,2 \\
\hline $\mathrm{C}$ & 25,581 & 7,712 & 3,955 & 0,12 & 0,00 & 0,01 & 11,3 & 19,2 & 4,2 & 1,4 & 1,6 & 0,3 \\
\hline C & 24,288 & 10,037 & 3,662 & $-0,01$ & 0,00 & 0,00 & 11,6 & 17,5 & 4,5 & $-0,7$ & 1,8 & $-0,1$ \\
\hline C & 25,534 & 12,403 & 3,964 & $-0,05$ & $-0,03$ & $-0,01$ & 8,5 & 16,9 & 5,8 & $-0,5$ & 0,7 & $-0,1$ \\
\hline C & 24,275 & 14,618 & 3,663 & $-0,04$ & $-0,05$ & 0,00 & 9,2 & 13,9 & 6,2 & $-1,7$ & 0,8 & $-0,4$ \\
\hline $\mathrm{C}$ & 28,229 & 7,733 & 4,501 & $-0,03$ & $-0,01$ & $-0,01$ & 9,1 & 4,9 & 4,4 & 0,4 & 1,0 & 0,1 \\
\hline $\mathrm{H}$ & 20,745 & $-1,004$ & 2,778 & 0,05 & 0,09 & 0,01 & 1,9 & 5,6 & 1,3 & 1,9 & 0,2 & 0,5 \\
\hline $\mathrm{H}$ & 18,425 & 3,051 & 2,172 & 0,08 & 0,03 & 0,02 & 4,5 & 2,9 & 1,3 & $-0,2$ & 0,9 & 0,0 \\
\hline $\mathrm{H}$ & 18,411 & 12,408 & 2,260 & 0,10 & $-0,02$ & 0,02 & 4,2 & 1,2 & 1,3 & $-0,8$ & 0,7 & $-0,2$ \\
\hline $\mathrm{H}$ & 20,696 & 16,411 & 2,775 & 0,05 & $-0,09$ & 0,01 & 1,9 & 5,5 & 1,3 & $-1,9$ & 0,2 & $-0,5$ \\
\hline $\mathrm{H}$ & 25,203 & $-1,004$ & 3,918 & $-0,05$ & 0,09 & $-0,01$ & 1,9 & 5,6 & 1,3 & $-1,9$ & 0,2 & $-0,5$ \\
\hline $\mathrm{H}$ & 27,523 & 3,051 & 4,524 & $-0,09$ & 0,02 & $-0,03$ & 4,1 & 1,2 & 1,3 & $-0,8$ & 0,8 & $-0,2$ \\
\hline $\mathrm{H}$ & 27,537 & 12,408 & 4,436 & $-0,08$ & $-0,03$ & $-0,02$ & 4,5 & 3,0 & 1,3 & $-0,2$ & 0,8 & 0,0 \\
\hline $\mathrm{H}$ & 25,252 & 16,411 & 3,921 & $-0,05$ & $-0,09$ & $-0,01$ & 1,9 & 5,5 & 1,3 & 2,0 & 0,2 & 0,5 \\
\hline $\mathrm{H}$ & 17,049 & 5,831 & 2,035 & 0,08 & 0,11 & 0,02 & 2,7 & 4,7 & 1,2 & 0,5 & 0,3 & 0,2 \\
\hline $\mathrm{H}$ & 17,396 & 8,709 & 0,454 & 0,05 & $-0,06$ & 0,12 & 2,3 & 1,5 & 3,6 & $-0,6$ & 1,5 & $-1,1$ \\
\hline $\mathrm{H}$ & 16,730 & 8,672 & 3,688 & 0,10 & $-0,06$ & $-0,09$ & 3,6 & 1,4 & 2,3 & $-1,0$ & $-1,6$ & 0,7 \\
\hline $\mathrm{H}$ & 28,872 & 9,644 & 4,653 & $-0,08$ & $-0,10$ & $-0,02$ & 2,6 & 4,7 & 1,2 & 0,4 & 0,3 & 0,1 \\
\hline
\end{tabular}




\begin{tabular}{ccccccccccccc}
\hline H & 29,232 & 6,801 & 3,012 & $-0,10$ & 0,06 & 0,09 & 3,6 & 1,4 & 2,3 & $-1,0$ & $-1,6$ & 0,7 \\
\hline H & 28,565 & 6,768 & 6,246 & $-0,05$ & 0,06 & $-0,12$ & 2,3 & 1,5 & 3,6 & $-0,6$ & 1,5 & $-1,0$ \\
\hline & & \multicolumn{7}{c}{ Ending Chain Toluene } \\
\hline C & 30,457 & 7,805 & 4,890 & 0,01 & 0,01 & 0,00 & 9,5 & 4,2 & 4,6 & 0,1 & 0,7 & $-0,2$ \\
\hline C & 33,137 & 7,925 & 5,306 & $-0,12$ & 0,01 & $-0,02$ & 12,2 & 9,3 & 5,6 & $-0,2$ & 0,9 & 2,3 \\
\hline C & 34,686 & 5,916 & 4,557 & 0,01 & 0,07 & 0,04 & 11,8 & 8,6 & 6,6 & $-1,5$ & 0,0 & 1,0 \\
\hline C & 37,267 & 6,053 & 4,944 & $-0,06$ & 0,05 & 0,01 & 10,8 & 8,9 & 6,8 & 0,8 & 1,0 & 1,4 \\
\hline C & 38,328 & 8,155 & 6,071 & $-0,09$ & $-0,01$ & $-0,01$ & 9,4 & 10,1 & 7,1 & $-0,3$ & 0,2 & 1,8 \\
\hline C & 36,806 & 10,158 & 6,807 & $-0,04$ & $-0,05$ & $-0,03$ & 11,3 & 8,7 & 6,6 & $-0,9$ & 0,3 & 1,1 \\
\hline C & 34,225 & 10,048 & 6,428 & 0,01 & $-0,08$ & $-0,03$ & 11,2 & 8,7 & 6,8 & 1,4 & 1,3 & 1,5 \\
\hline H & 33,840 & 4,258 & 3,680 & 0,04 & 0,09 & 0,05 & 1,6 & 3,9 & 2,0 & 1,2 & 0,6 & 1,4 \\
\hline H & 38,422 & 4,466 & 4,326 & $-0,07$ & 0,08 & 0,03 & 2,6 & 3,6 & 1,6 & $-1,9$ & $-0,7$ & 0,9 \\
\hline H & 40,361 & 8,205 & 6,389 & $-0,11$ & $-0,01$ & $-0,02$ & 5,4 & 1,2 & 1,4 & 0,2 & 0,7 & 0,0 \\
\hline H & 37,626 & 11,823 & 7,695 & $-0,05$ & $-0,09$ & $-0,05$ & 1,9 & 3,9 & 2,0 & 1,4 & 0,8 & 1,4 \\
\hline H & 32,975 & 11,585 & 6,985 & 0,07 & $-0,09$ & $-0,03$ & 2,4 & 3,6 & 1,6 & $-1,7$ & $-0,7$ & 0,9 \\
\hline H & 29,989 & 6,043 & 4,017 & 0,06 & 0,13 & 0,07 & 2,2 & 3,1 & 1,8 & 1,3 & 0,7 & 1,0 \\
\hline H & 29,894 & 9,326 & 3,683 & 0,07 & $-0,10$ & 0,08 & 2,6 & 2,6 & 2,4 & $-1,2$ & 1,1 & $-1,3$ \\
\hline H & 29,493 & 7,958 & 6,661 & 0,09 & $-0,01$ & $-0,12$ & 3,3 & 1,2 & 3,1 & $-0,1$ & $-1,8$ & 0,1 \\
\hline
\end{tabular}


Table S20: atomic coordinates, dipole moments and polarizabilities of the polyaromatic molecule calculated with QTAIM, all values in a.u.

\begin{tabular}{|c|c|c|c|c|c|c|c|c|c|c|c|c|}
\hline Atom & $\mathbf{x}$ & $\mathbf{y}$ & $\mathbf{z}$ & $\mu_{\mathrm{x}}$ & $\mu_{y}$ & $\mu_{\mathrm{z}}$ & $\alpha_{x x}$ & $\alpha_{\mathrm{yy}}$ & $\alpha_{\mathrm{zz}}$ & $\alpha_{\mathrm{xy}}$ & $\alpha_{\mathrm{xz}}$ & $\alpha_{\mathrm{yz}}$ \\
\hline $\mathrm{C}$ & 21,680 & 0,814 & 3,011 & 0,05 & 0,03 & 0,02 & 10,2 & 13,3 & 6,0 & $-2,4$ & 1,1 & $-0,5$ \\
\hline C & 20,415 & 3,023 & 2,695 & 0,03 & $-0,01$ & 0,00 & 8,0 & 16,9 & 5,5 & $-1,0$ & 0,7 & $-0,1$ \\
\hline C & 21,661 & 5,391 & 3,031 & 0,02 & $-0,08$ & 0,00 & 20,5 & 18,8 & 4,6 & $-4,9$ & 3,7 & $-0,8$ \\
\hline $\mathrm{C}$ & 20,367 & 7,712 & 2,741 & 0,10 & 0,00 & 0,03 & 30,6 & 19,4 & 4,9 & $-0,3$ & 5,5 & 0,0 \\
\hline $\mathrm{C}$ & 21,660 & 10,037 & 3,035 & 0,02 & 0,08 & 0,01 & 20,4 & 18,5 & 4,5 & 4,9 & 3,7 & 0,9 \\
\hline C & 20,414 & 12,403 & 2,732 & 0,03 & 0,01 & 0,01 & 7,9 & 16,7 & 5,4 & 1,0 & 0,6 & 0,2 \\
\hline $\mathrm{C}$ & 21,673 & 14,618 & 3,033 & 0,05 & $-0,04$ & 0,01 & 10,2 & 13,1 & 6,0 & 2,3 & 1,0 & 0,5 \\
\hline $\mathrm{C}$ & 17,718 & 7,732 & 2,195 & 0,12 & 0,02 & 0,04 & 42,9 & 4,0 & 6,6 & $-0,8$ & 7,0 & $-0,1$ \\
\hline $\mathrm{C}$ & 15,490 & 7,805 & 1,806 & $-0,17$ & 0,01 & $-0,02$ & 41,6 & 4,3 & 6,6 & $-1,1$ & 6,3 & 0,1 \\
\hline C & 12,811 & 7,925 & 1,390 & $-0,17$ & 0,01 & $-0,02$ & 26,9 & 8,6 & 5,8 & $-0,2$ & 3,4 & $-2,3$ \\
\hline C & 11,262 & 5,916 & 2,139 & $-0,06$ & 0,00 & $-0,01$ & 18,4 & 7,4 & 5,6 & 3,7 & 0,0 & $-0,5$ \\
\hline C & 8,681 & 6,053 & 1,753 & 0,02 & 0,06 & $-0,02$ & 15,6 & 8,3 & 6,8 & $-1,0$ & 1,9 & $-1,4$ \\
\hline $\mathrm{C}$ & 7,620 & 8,154 & 0,625 & 0,05 & $-0,01$ & 0,01 & 13,3 & 9,3 & 6,8 & 0,2 & 1,0 & $-1,7$ \\
\hline $\mathrm{C}$ & 9,142 & 10,158 & $-0,111$ & 0,00 & $-0,05$ & 0,02 & 16,2 & 8,1 & 6,5 & 0,8 & 1,0 & $-1,1$ \\
\hline C & 11,723 & 10,048 & 0,268 & $-0,06$ & 0,00 & $-0,01$ & 16,7 & 7,7 & 6,8 & $-4,0$ & 3,6 & $-1,9$ \\
\hline C & 24,267 & 0,814 & 3,685 & $-0,05$ & 0,03 & $-0,02$ & 10,2 & 13,3 & 6,0 & 2,4 & 1,1 & 0,5 \\
\hline $\mathrm{C}$ & 25,533 & 3,023 & 4,001 & $-0,03$ & $-0,01$ & 0,00 & 8,0 & 16,9 & 5,5 & 1,0 & 0,7 & 0,1 \\
\hline C & 24,287 & 5,391 & 3,665 & $-0,02$ & $-0,08$ & 0,00 & 20,5 & 18,8 & 4,6 & 4,9 & 3,7 & 0,8 \\
\hline $\mathrm{C}$ & 25,581 & 7,712 & 3,955 & $-0,10$ & 0,00 & $-0,03$ & 30,6 & 19,4 & 4,8 & 0,3 & 5,5 & 0,0 \\
\hline C & 24,288 & 10,037 & 3,662 & $-0,02$ & 0,08 & $-0,01$ & 20,4 & 18,5 & 4,5 & $-4,9$ & 3,7 & $-0,9$ \\
\hline $\mathrm{C}$ & 25,534 & 12,403 & 3,964 & $-0,03$ & 0,01 & $-0,01$ & 7,8 & 16,7 & 5,4 & $-1,0$ & 0,6 & $-0,2$ \\
\hline $\mathrm{C}$ & 24,275 & 14,618 & 3,663 & $-0,05$ & $-0,04$ & $-0,01$ & 10,2 & 13,1 & 6,0 & $-2,3$ & 1,0 & $-0,5$ \\
\hline $\mathrm{C}$ & 28,229 & 7,732 & 4,501 & $-0,12$ & 0,02 & $-0,04$ & 42,9 & 4,0 & 6,6 & 0,8 & 7,1 & 0,1 \\
\hline C & 30,457 & 7,805 & 4,890 & 0,17 & 0,01 & 0,02 & 41,5 & 4,3 & 6,6 & 1,1 & 6,3 & $-0,1$ \\
\hline C & 33,137 & 7,925 & 5,306 & 0,17 & 0,01 & 0,02 & 26,9 & 8,6 & 5,8 & 0,2 & 3,5 & 2,3 \\
\hline C & 34,686 & 5,916 & 4,557 & 0,06 & 0,00 & 0,01 & 18,3 & 7,4 & 5,6 & $-3,8$ & 0,0 & 0,5 \\
\hline C & 37,267 & 6,053 & 4,944 & $-0,02$ & 0,06 & 0,02 & 15,6 & 8,3 & 6,7 & 1,0 & 1,9 & 1,4 \\
\hline C & 38,328 & 8,154 & 6,071 & $-0,06$ & $-0,01$ & $-0,01$ & 13,2 & 9,2 & 6,8 & $-0,2$ & 0,9 & 1,7 \\
\hline C & 36,806 & 10,158 & 6,807 & 0,00 & $-0,05$ & $-0,02$ & 16,1 & 8,1 & 6,5 & $-0,8$ & 1,0 & 1,1 \\
\hline C & 34,225 & 10,048 & 6,428 & 0,06 & 0,00 & 0,01 & 16,7 & 7,7 & 6,8 & 3,9 & 3,6 & 2,0 \\
\hline $\mathrm{H}$ & 20,745 & $-1,004$ & 2,778 & 0,05 & 0,09 & 0,01 & 1,8 & 5,4 & 1,3 & 1,8 & 0,2 & 0,5 \\
\hline $\mathrm{H}$ & 18,425 & 3,051 & 2,172 & 0,08 & 0,01 & 0,02 & 3,9 & 1,1 & 1,2 & 0,5 & 0,8 & 0,2 \\
\hline $\mathrm{H}$ & 18,411 & 12,408 & 2,260 & 0,08 & $-0,01$ & 0,02 & 4,0 & 1,1 & 1,2 & $-0,5$ & 0,7 & $-0,1$ \\
\hline $\mathrm{H}$ & 20,696 & 16,411 & 2,775 & 0,05 & $-0,09$ & 0,01 & 1,8 & 5,3 & 1,3 & $-1,8$ & 0,1 & $-0,5$ \\
\hline $\mathrm{H}$ & 12,108 & 4,258 & 3,016 & $-0,03$ & 0,08 & $-0,04$ & 1,5 & 3,2 & 1,7 & $-0,6$ & 0,3 & $-1,1$ \\
\hline $\mathrm{H}$ & 7,526 & 4,466 & 2,370 & 0,06 & 0,07 & $-0,03$ & 3,4 & 3,3 & 1,5 & 2,3 & $-0,8$ & $-0,7$ \\
\hline $\mathrm{H}$ & 5,587 & 8,205 & 0,307 & 0,10 & $-0,01$ & 0,02 & 6,8 & 1,1 & 1,4 & $-0,2$ & 0,9 & 0,0 \\
\hline $\mathrm{H}$ & 8,322 & 11,823 & $-0,999$ & 0,04 & $-0,08$ & 0,04 & 2,4 & 3,6 & 2,1 & $-1,9$ & 1,1 & $-1,4$ \\
\hline $\mathrm{H}$ & 12,973 & 11,585 & $-0,289$ & $-0,05$ & $-0,07$ & 0,03 & 2,0 & 2,9 & 1,5 & 1,0 & $-0,4$ & $-0,8$ \\
\hline $\mathrm{H}$ & 25,203 & $-1,004$ & 3,918 & $-0,05$ & 0,09 & $-0,01$ & 1,8 & 5,4 & 1,3 & $-1,8$ & 0,2 & $-0,5$ \\
\hline $\mathrm{H}$ & 27,523 & 3,051 & 4,524 & $-0,08$ & 0,01 & $-0,02$ & 3,9 & 1,1 & 1,2 & $-0,5$ & 0,8 & $-0,2$ \\
\hline $\mathrm{H}$ & 27,537 & 12,408 & 4,436 & $-0,08$ & $-0,01$ & $-0,02$ & 4,0 & 1,1 & 1,2 & 0,5 & 0,7 & 0,1 \\
\hline $\mathrm{H}$ & 25,252 & 16,411 & 3,921 & $-0,05$ & $-0,09$ & $-0,01$ & 1,8 & 5,3 & 1,3 & 1,8 & 0,1 & 0,5 \\
\hline $\mathrm{H}$ & 33,840 & 4,258 & 3,680 & 0,03 & 0,08 & 0,04 & 1,5 & 3,2 & 1,7 & 0,6 & 0,3 & 1,1 \\
\hline $\mathrm{H}$ & 38,422 & 4,466 & 4,326 & $-0,06$ & 0,07 & 0,03 & 3,4 & 3,3 & 1,5 & $-2,3$ & $-0,8$ & 0,7 \\
\hline
\end{tabular}




\begin{tabular}{ccccccccccccc}
\hline $\mathbf{H}$ & 40,361 & 8,205 & 6,389 & $-0,10$ & $-0,01$ & $-0,02$ & 6,8 & 1,1 & 1,4 & 0,2 & 0,9 & 0,0 \\
\hline $\mathbf{H}$ & 37,626 & 11,823 & 7,695 & $-0,04$ & $-0,08$ & $-0,04$ & 2,4 & 3,6 & 2,1 & 1,9 & 1,1 & 1,4 \\
\hline $\mathbf{H}$ & 32,975 & 11,585 & 6,985 & 0,05 & $-0,07$ & $-0,03$ & 2,0 & 2,9 & 1,5 & $-1,0$ & $-0,4$ & 0,8 \\
\hline
\end{tabular}


Table S21: atomic coordinates, dipole moments and polarizabilities of the polyaromatic molecule calculated with ADIM, all values in a.u.

\begin{tabular}{|c|c|c|c|c|c|c|c|c|c|c|c|c|}
\hline Atom & $X$ & y & $\mathbf{z}$ & $\mu_{\mathrm{x}}$ & $\mu_{y}$ & $\mu_{\mathrm{z}}$ & $\alpha_{x x}$ & $\alpha_{y y}$ & $\alpha_{\mathrm{zz}}$ & $\alpha_{\mathrm{xy}}$ & $\alpha_{x z}$ & $\alpha_{y z}$ \\
\hline $\mathrm{C}$ & 15,490 & 7,805 & 1,806 & 0,00 & $-0,01$ & 0,00 & 29,1 & 1,6 & 3,0 & $-1,7$ & 5,2 & 0,1 \\
\hline C & 12,811 & 7,925 & 1,390 & 0,11 & 0,00 & 0,02 & 16,5 & 7,7 & 5,1 & $-0,4$ & 1,9 & $-1,9$ \\
\hline C & 11,262 & 5,916 & 2,139 & 0,00 & 0,07 & $-0,04$ & 14,5 & 7,6 & 6,0 & 2,1 & 0,5 & $-0,9$ \\
\hline $\mathrm{C}$ & 8,681 & 6,053 & 1,753 & 0,05 & 0,05 & $-0,01$ & 12,2 & 8,2 & 6,6 & $-0,8$ & 1,2 & $-1,3$ \\
\hline C & 7,620 & 8,155 & 0,625 & 0,08 & $-0,01$ & 0,01 & 10,5 & 9,6 & 7,0 & 0,3 & 0,5 & $-1,7$ \\
\hline C & 9,142 & 10,158 & $-0,111$ & 0,04 & $-0,05$ & 0,03 & 12,5 & 8,1 & 6,5 & 0,7 & 0,6 & $-1,1$ \\
\hline $\mathrm{C}$ & 11,723 & 10,048 & 0,268 & $-0,02$ & $-0,08$ & 0,03 & 13,7 & 7,9 & 6,7 & $-2,3$ & 2,0 & $-1,4$ \\
\hline $\mathrm{H}$ & 12,108 & 4,258 & 3,016 & $-0,05$ & 0,09 & $-0,05$ & 2,0 & 3,3 & 1,8 & $-1,2$ & 0,6 & $-1,2$ \\
\hline $\mathrm{H}$ & 7,526 & 4,466 & 2,370 & 0,07 & 0,08 & $-0,03$ & 2,9 & 3,5 & 1,5 & 2,2 & $-0,7$ & $-0,8$ \\
\hline $\mathrm{H}$ & 5,587 & 8,205 & 0,307 & 0,11 & $-0,01$ & 0,02 & 5,8 & 1,1 & 1,3 & $-0,2$ & 0,7 & 0,0 \\
\hline $\mathrm{H}$ & 8,322 & 11,823 & $-0,999$ & 0,05 & $-0,09$ & 0,05 & 2,1 & 3,8 & 2,0 & $-1,7$ & 0,8 & $-1,4$ \\
\hline $\mathrm{H}$ & 12,973 & 11,585 & $-0,289$ & $-0,06$ & $-0,09$ & 0,03 & 2,6 & 3,0 & 1,5 & 1,6 & $-0,5$ & $-0,8$ \\
\hline C & 30,457 & 7,805 & 4,890 & 0,00 & 0,01 & 0,00 & 29,1 & 1,6 & 2,9 & 0,4 & 5,1 & 0,1 \\
\hline C & 33,137 & 7,925 & 5,306 & $-0,11$ & 0,01 & $-0,02$ & 16,9 & 7,7 & 5,2 & 0,0 & 2,0 & 1,9 \\
\hline C & 34,686 & 5,916 & 4,557 & 0,01 & 0,07 & 0,04 & 14,5 & 7,7 & 6,2 & $-2,2$ & 0,5 & 0,8 \\
\hline C & 37,267 & 6,053 & 4,944 & $-0,06$ & 0,05 & 0,01 & 12,2 & 8,2 & 6,6 & 0,7 & 1,2 & 1,3 \\
\hline C & 38,328 & 8,155 & 6,071 & $-0,08$ & $-0,01$ & $-0,01$ & 10,4 & 9,5 & 7,0 & $-0,3$ & 0,5 & 1,7 \\
\hline C & 36,806 & 10,158 & 6,807 & $-0,04$ & $-0,05$ & $-0,03$ & 12,6 & 8,1 & 6,4 & $-0,7$ & 0,7 & 1,1 \\
\hline $\mathrm{C}$ & 34,225 & 10,048 & 6,428 & 0,01 & $-0,08$ & $-0,03$ & 13,7 & 7,9 & 6,6 & 2,2 & 2,1 & 1,5 \\
\hline $\mathrm{H}$ & 33,840 & 4,258 & 3,680 & 0,04 & 0,09 & 0,05 & 1,9 & 3,3 & 1,8 & 1,1 & 0,6 & 1,2 \\
\hline $\mathrm{H}$ & 38,422 & 4,466 & 4,326 & $-0,07$ & 0,08 & 0,03 & 2,9 & 3,5 & 1,5 & $-2,2$ & $-0,7$ & 0,8 \\
\hline $\mathrm{H}$ & 40,361 & 8,205 & 6,389 & $-0,11$ & $-0,01$ & $-0,02$ & 5,8 & 1,1 & 1,3 & 0,2 & 0,7 & 0,0 \\
\hline $\mathrm{H}$ & 37,626 & 11,823 & 7,695 & $-0,05$ & $-0,09$ & $-0,05$ & 2,1 & 3,7 & 2,0 & 1,7 & 0,8 & 1,4 \\
\hline $\mathrm{H}$ & 32,975 & 11,585 & 6,985 & 0,07 & $-0,09$ & $-0,03$ & 2,7 & 3,0 & 1,5 & $-1,6$ & $-0,5$ & 0,8 \\
\hline C & 21,680 & 0,814 & 3,011 & 0,05 & 0,04 & 0,02 & 9,9 & 13,9 & 6,0 & $-2,3$ & 1,1 & $-0,4$ \\
\hline $\mathrm{C}$ & 20,415 & 3,023 & 2,695 & 0,05 & 0,02 & 0,01 & 9,8 & 16,5 & 5,5 & $-2,0$ & 1,1 & $-0,1$ \\
\hline C & 21,661 & 5,391 & 3,031 & 0,02 & $-0,01$ & 0,00 & 14,1 & 16,4 & 4,4 & $-1,5$ & 2,3 & $-0,1$ \\
\hline C & 20,367 & 7,712 & 2,741 & $-0,12$ & 0,01 & $-0,01$ & 15,5 & 16,9 & 4,0 & 1,7 & 2,4 & 0,2 \\
\hline $\mathrm{C}$ & 21,660 & 10,037 & 3,035 & 0,02 & 0,00 & 0,00 & 14,2 & 18,1 & 4,2 & 2,6 & 2,2 & 0,3 \\
\hline C & 20,414 & 12,403 & 2,732 & 0,06 & $-0,01$ & 0,02 & 8,9 & 16,7 & 5,5 & 1,1 & 0,7 & 0,0 \\
\hline $\mathrm{C}$ & 21,673 & 14,618 & 3,033 & 0,05 & $-0,04$ & 0,01 & 10,0 & 13,3 & 6,0 & 1,8 & 0,9 & 0,4 \\
\hline $\mathrm{C}$ & 17,718 & 7,733 & 2,195 & 0,04 & 0,01 & 0,01 & 27,9 & 2,3 & 3,0 & 0,8 & 4,4 & $-0,2$ \\
\hline C & 24,267 & 0,814 & 3,685 & $-0,05$ & 0,04 & $-0,02$ & 9,8 & 13,4 & 6,0 & 1,9 & 1,0 & 0,3 \\
\hline $\mathrm{C}$ & 25,533 & 3,023 & 4,001 & $-0,06$ & 0,02 & $-0,01$ & 8,9 & 16,9 & 5,5 & 1,1 & 0,8 & $-0,1$ \\
\hline $\mathrm{C}$ & 24,287 & 5,391 & 3,665 & $-0,02$ & 0,00 & 0,00 & 14,1 & 18,3 & 4,3 & 2,7 & 2,3 & 0,2 \\
\hline C & 25,581 & 7,712 & 3,955 & 0,12 & $-0,01$ & 0,01 & 15,5 & 16,9 & 4,0 & 2,1 & 2,4 & 0,3 \\
\hline $\mathrm{C}$ & 24,288 & 10,037 & 3,662 & $-0,01$ & 0,01 & 0,00 & 14,2 & 16,2 & 4,3 & $-1,5$ & 2,2 & $-0,2$ \\
\hline $\mathrm{C}$ & 25,534 & 12,403 & 3,964 & $-0,05$ & $-0,02$ & $-0,01$ & 9,8 & 16,3 & 5,5 & $-2,0$ & 0,9 & $-0,2$ \\
\hline C & 24,275 & 14,618 & 3,663 & $-0,05$ & $-0,05$ & 0,00 & 10,0 & 13,7 & 5,9 & $-2,3$ & 0,9 & $-0,5$ \\
\hline C & 28,229 & 7,733 & 4,501 & $-0,05$ & $-0,01$ & $-0,01$ & 28,0 & 2,4 & 2,9 & 1,7 & 4,2 & 0,2 \\
\hline $\mathrm{H}$ & 20,745 & $-1,004$ & 2,778 & 0,05 & 0,09 & 0,01 & 1,9 & 5,6 & 1,3 & 1,8 & 0,2 & 0,5 \\
\hline $\mathrm{H}$ & 18,425 & 3,051 & 2,172 & 0,08 & 0,03 & 0,02 & 5,2 & 3,0 & 1,3 & $-0,8$ & 1,0 & $-0,1$ \\
\hline $\mathrm{H}$ & 18,411 & 12,408 & 2,260 & 0,10 & $-0,01$ & 0,02 & 4,7 & 1,1 & 1,2 & $-0,7$ & 0,8 & $-0,1$ \\
\hline $\mathrm{H}$ & 20,696 & 16,411 & 2,775 & 0,05 & $-0,09$ & 0,01 & 1,9 & 5,5 & 1,3 & $-1,9$ & 0,2 & $-0,5$ \\
\hline $\mathrm{H}$ & 25,203 & $-1,004$ & 3,918 & $-0,05$ & 0,09 & $-0,01$ & 1,9 & 5,6 & 1,3 & $-1,8$ & 0,2 & $-0,5$ \\
\hline
\end{tabular}




\begin{tabular}{ccccccccccccc}
\hline $\mathbf{H}$ & 27,523 & 3,051 & 4,524 & $-0,09$ & 0,02 & $-0,03$ & 4,6 & 1,1 & 1,2 & $-0,7$ & 0,9 & $-0,1$ \\
\hline $\mathbf{H}$ & 27,537 & 12,408 & 4,436 & $-0,08$ & $-0,03$ & $-0,02$ & 5,3 & 2,9 & 1,2 & $-0,8$ & 0,9 & $-0,2$ \\
\hline $\mathbf{H}$ & 25,252 & 16,411 & 3,921 & $-0,05$ & $-0,09$ & $-0,01$ & 1,9 & 5,5 & 1,3 & 1,9 & 0,2 & 0,5 \\
\hline
\end{tabular}




\section{Five and seven-molecules benzene aggregates}

Figure S1: five-molecules benzene clusters obtained by totally quantum calculations (QTAIM) at M06-HF/aug-cc-pVDZ level of theory (on the left) and ADIM (on the right)
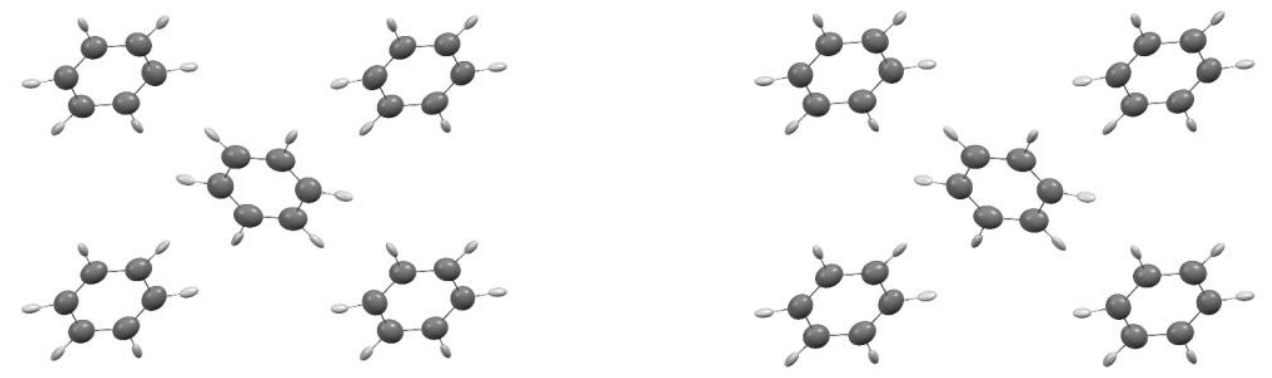

Table S22: atomic coordinates, dipole moments and polarizabilities of the central benzene within a five-molecule cluster using QTAIM, all values in a.u.

\begin{tabular}{|c|c|c|c|c|c|c|c|c|c|c|c|c|}
\hline Atom & $\mathbf{x}$ & $y$ & $\mathbf{z}$ & $\mu_{x}$ & $\mu_{y}$ & $\mu_{\mathrm{z}}$ & $\alpha_{x x}$ & $\alpha_{y y}$ & $\alpha_{\mathrm{zz}}$ & $\alpha_{x y}$ & $\alpha_{\mathrm{xz}}$ & $\alpha_{\mathrm{yz}}$ \\
\hline C & $-0,739$ & 2,477 & 0,123 & 0,01 & $-0,07$ & 0,02 & 6,6 & 10,7 & 9,9 & 0,1 & 1,9 & 0,4 \\
\hline $\mathrm{H}$ & $-1,212$ & 4,452 & 0,460 & 0,04 & $-0,08$ & 0,01 & 1,0 & 5,1 & 1,7 & $-1,0$ & $-0,3$ & 0,9 \\
\hline C & 1,157 & 1,606 & 1,735 & $-0,01$ & $-0,02$ & $-0,06$ & 6,6 & 12,6 & 8,2 & $-1,6$ & 1,6 & 0,1 \\
\hline $\mathrm{H}$ & 2,091 & 2,946 & 2,987 & $-0,05$ & $-0,04$ & $-0,05$ & 1,7 & 3,4 & 2,8 & 0,8 & 0,7 & 2,0 \\
\hline C & $-1,849$ & 0,906 & $-1,561$ & 0,06 & $-0,02$ & 0,06 & 6,2 & 13,1 & 8,0 & $-0,4$ & 1,3 & 1,1 \\
\hline $\mathrm{H}$ & $-3,338$ & 1,517 & $-2,844$ & 0,08 & $-0,05$ & 0,07 & 3,0 & 2,3 & 3,0 & $-0,7$ & 1,3 & $-1,4$ \\
\hline C & 0,739 & $-2,477$ & $-0,123$ & $-0,01$ & 0,07 & $-0,03$ & 6,6 & 10,7 & 10,0 & 0,1 & 1,9 & 0,4 \\
\hline $\mathrm{H}$ & 1,212 & $-4,452$ & $-0,460$ & $-0,04$ & 0,08 & $-0,01$ & 1,0 & 5,1 & 1,9 & $-1,0$ & $-0,3$ & 1,0 \\
\hline C & $-1,157$ & $-1,606$ & $-1,735$ & 0,01 & 0,02 & 0,06 & 6,6 & 12,7 & 8,3 & $-1,6$ & 1,6 & 0,2 \\
\hline $\mathrm{H}$ & $-2,091$ & $-2,946$ & $-2,987$ & 0,05 & 0,04 & 0,05 & 1,7 & 3,4 & 2,9 & 0,8 & 0,7 & 2,1 \\
\hline C & 1,849 & $-0,906$ & 1,561 & $-0,06$ & 0,02 & $-0,06$ & 6,2 & 13,1 & 8,2 & $-0,3$ & 1,4 & 1,1 \\
\hline $\mathrm{H}$ & 3,338 & $-1,517$ & 2,844 & $-0,08$ & 0,05 & $-0,07$ & 3,0 & 2,3 & 3,1 & $-0,7$ & 1,2 & $-1,6$ \\
\hline
\end{tabular}

Table S23: atomic coordinates, dipole moments and polarizabilities of the central benzene within a five-molecule cluster using ADIM, all values in a.u.

\begin{tabular}{ccccccccccccc}
\hline Atom & $\mathbf{x}$ & $\mathbf{y}$ & $\mathbf{z}$ & $\boldsymbol{\mu}_{\mathbf{x}}$ & $\boldsymbol{\mu}_{\mathbf{y}}$ & $\boldsymbol{\mu}_{\mathbf{z}}$ & $\boldsymbol{\alpha}_{\mathbf{x x}}$ & $\boldsymbol{\alpha}_{\mathrm{yy}}$ & $\boldsymbol{\alpha}_{\mathrm{zz}}$ & $\boldsymbol{\alpha}_{\mathbf{x y}}$ & $\boldsymbol{\alpha}_{\mathrm{xz}}$ & $\boldsymbol{\alpha}_{\mathrm{yz}}$ \\
\hline $\mathbf{C}$ & $-0,739$ & 2,477 & 0,123 & 0,01 & $-0,09$ & 0,01 & 6,2 & 10,0 & 9,3 & 0,1 & 1,8 & 0,7 \\
\hline $\mathrm{H}$ & $-1,212$ & 4,452 & 0,460 & 0,04 & $-0,12$ & 0,00 & 1,0 & 4,9 & 1,6 & $-0,5$ & $-0,6$ & 0,4 \\
\hline $\mathbf{C}$ & 1,157 & 1,606 & 1,735 & $-0,01$ & $-0,05$ & $-0,07$ & 6,5 & 12,0 & 8,2 & $-1,2$ & 1,1 & 0,2 \\
\hline $\mathrm{H}$ & 2,091 & 2,946 & 2,987 & $-0,05$ & $-0,07$ & $-0,08$ & 1,5 & 3,1 & 2,8 & 0,6 & 1,0 & 2,0 \\
\hline $\mathbf{C}$ & $-1,849$ & 0,906 & $-1,561$ & 0,06 & $-0,02$ & 0,05 & 5,8 & 12,4 & 7,7 & 0,1 & 0,9 & 1,1 \\
\hline $\mathrm{H}$ & $-3,338$ & 1,517 & $-2,844$ & 0,08 & $-0,04$ & 0,06 & 2,8 & 1,9 & 2,6 & $-0,7$ & 1,5 & $-1,2$ \\
\hline $\mathbf{C}$ & 0,739 & $-2,477$ & $-0,123$ & $-0,01$ & 0,09 & $-0,01$ & 6,2 & 10,0 & 9,3 & 0,1 & 1,8 & 0,6 \\
\hline $\mathrm{H}$ & 1,212 & $-4,452$ & $-0,460$ & $-0,04$ & 0,12 & 0,00 & 1,0 & 4,9 & 1,6 & $-0,5$ & $-0,6$ & 0,4 \\
\hline $\mathbf{C}$ & $-1,157$ & $-1,606$ & $-1,735$ & 0,01 & 0,05 & 0,07 & 6,5 & 12,0 & 8,1 & $-1,2$ & 1,0 & 0,2 \\
\hline $\mathrm{H}$ & $-2,091$ & $-2,946$ & $-2,987$ & 0,05 & 0,07 & 0,08 & 1,5 & 3,1 & 2,8 & 0,6 & 1,0 & 2,0 \\
\hline $\mathrm{C}$ & 1,849 & $-0,906$ & 1,561 & $-0,06$ & 0,02 & $-0,05$ & 5,8 & 12,4 & 7,7 & 0,1 & 0,9 & 1,1 \\
\hline $\mathrm{H}$ & 3,338 & $-1,517$ & 2,844 & $-0,08$ & 0,04 & $-0,06$ & 2,8 & 1,9 & 2,6 & $-0,7$ & 1,5 & $-1,2$ \\
\hline
\end{tabular}


Figure S2: seven-molecules benzene clusters obtained by totally quantum calculations (QTAIM) at M06-HF/aug-cc-pVDZ level of theory (on the left) and ADIM (on the right)
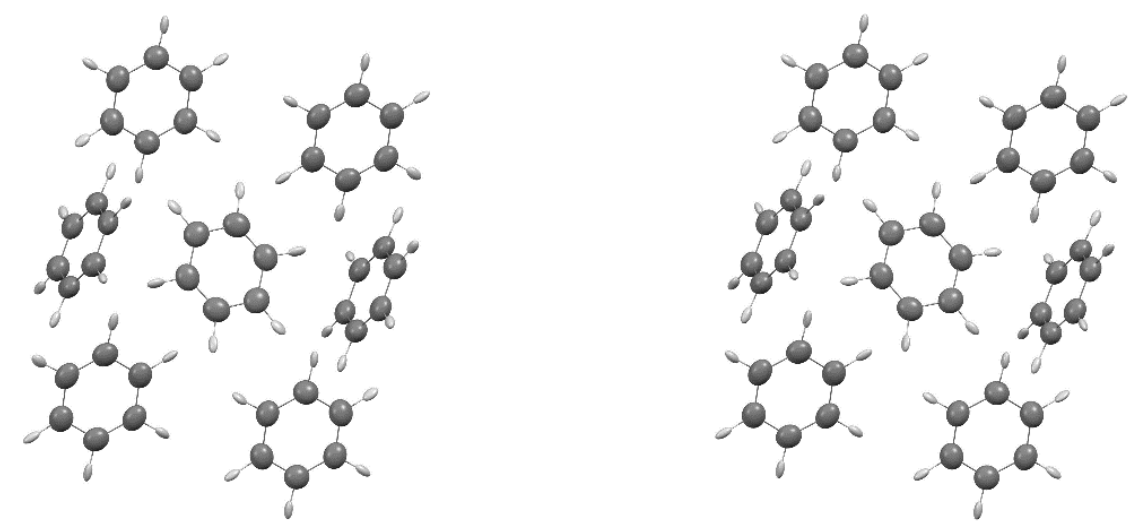

Table S24: atomic coordinates, dipole moments and polarizabilities of the central benzene within a seven-molecule cluster using QTAIM, all values in a.u.

\begin{tabular}{|c|c|c|c|c|c|c|c|c|c|c|c|c|}
\hline Atom & $\mathrm{x}$ & $y$ & $\mathbf{z}$ & $\mu_{\mathrm{x}}$ & $\mu_{y}$ & $\mu_{\mathrm{z}}$ & $\alpha_{x x}$ & $\alpha_{y y}$ & $\alpha_{\mathrm{zz}}$ & $\alpha_{x y}$ & $\alpha_{\mathrm{xz}}$ & $\alpha_{y z}$ \\
\hline $\mathrm{C}$ & $-0,739$ & 2,477 & 0,123 & 0,01 & $-0,08$ & 0,01 & 7,3 & 9,6 & 10,3 & 0,1 & 3,2 & 0,4 \\
\hline $\mathrm{H}$ & $-1,212$ & 4,452 & 0,460 & 0,04 & $-0,09$ & 0,01 & 1,0 & 4,7 & 1,7 & $-0,8$ & $-0,1$ & 0,8 \\
\hline C & 1,157 & 1,606 & 1,735 & 0,00 & $-0,03$ & $-0,06$ & 7,3 & 10,7 & 8,5 & $-1,5$ & 2,9 & $-0,1$ \\
\hline $\mathrm{H}$ & 2,091 & 2,946 & 2,987 & $-0,04$ & $-0,05$ & $-0,04$ & 2,4 & 2,8 & 3,0 & 0,6 & 1,5 & 1,6 \\
\hline C & $-1,849$ & 0,906 & $-1,561$ & 0,05 & $-0,02$ & 0,05 & 6,4 & 11,2 & 8,3 & 0,1 & 2,5 & 1,2 \\
\hline $\mathrm{H}$ & $-3,338$ & 1,517 & $-2,844$ & 0,06 & $-0,04$ & 0,04 & 2,9 & 1,8 & 3,3 & $-0,6$ & 2,0 & $-1,2$ \\
\hline C & 0,739 & $-2,477$ & $-0,123$ & $-0,01$ & 0,08 & $-0,02$ & 7,4 & 9,7 & 10,4 & 0,1 & 3,1 & 0,3 \\
\hline $\mathrm{H}$ & 1,212 & $-4,452$ & $-0,460$ & $-0,04$ & 0,09 & $-0,01$ & 1,0 & 4,7 & 1,7 & $-0,8$ & $-0,2$ & 0,8 \\
\hline C & $-1,157$ & $-1,606$ & $-1,735$ & 0,00 & 0,03 & 0,06 & 7,3 & 10,8 & 8,6 & $-1,5$ & 2,9 & 0,0 \\
\hline $\mathrm{H}$ & $-2,091$ & $-2,946$ & $-2,987$ & 0,04 & 0,05 & 0,04 & 2,5 & 2,8 & 3,1 & 0,5 & 1,6 & 1,6 \\
\hline C & 1,849 & $-0,906$ & 1,561 & $-0,05$ & 0,02 & $-0,05$ & 6,4 & 11,1 & 8,3 & 0,1 & 2,5 & 1,2 \\
\hline $\mathrm{H}$ & 3,338 & $-1,517$ & 2,844 & $-0,06$ & 0,04 & $-0,04$ & 2,9 & 1,8 & 3,2 & $-0,6$ & 1,9 & $-1,2$ \\
\hline
\end{tabular}

Table S25: atomic coordinates, dipole moments and polarizabilities of the central benzene within a seven-molecule cluster using ADIM, all values in a.u

\begin{tabular}{|c|c|c|c|c|c|c|c|c|c|c|c|c|}
\hline Atom & $\mathrm{x}$ & $\mathbf{y}$ & $\mathbf{z}$ & $\mu_{x}$ & $\mu_{y}$ & $\mu_{\mathrm{z}}$ & $\alpha_{x x}$ & $\alpha_{y y}$ & $\alpha_{\mathrm{zz}}$ & $\alpha_{x y}$ & $\alpha_{\mathrm{xz}}$ & $\alpha_{y z}$ \\
\hline C & $-0,739$ & 2,477 & 0,123 & 0,02 & $-0,08$ & 0,02 & 6,7 & 9,0 & 9,6 & 0,3 & 2,9 & 0,6 \\
\hline $\mathrm{H}$ & $-1,212$ & 4,452 & 0,460 & 0,04 & $-0,11$ & 0,00 & 1,0 & 4,6 & 1,6 & $-0,5$ & $-0,4$ & 0,4 \\
\hline C & 1,157 & 1,606 & 1,735 & $-0,02$ & $-0,04$ & $-0,07$ & 7,1 & 10,5 & 8,4 & $-1,3$ & 2,5 & $-0,1$ \\
\hline $\mathrm{H}$ & 2,091 & 2,946 & 2,987 & $-0,05$ & $-0,07$ & $-0,08$ & 1,8 & 2,3 & 2,9 & 1,0 & 1,4 & 1,3 \\
\hline C & $-1,849$ & 0,906 & $-1,561$ & 0,08 & $-0,01$ & 0,07 & 6,3 & 10,8 & 8,1 & 0,7 & 2,2 & 1,1 \\
\hline $\mathrm{H}$ & $-3,338$ & 1,517 & $-2,844$ & 0,10 & $-0,05$ & 0,08 & 3,2 & 1,4 & 3,0 & $-0,8$ & 2,3 & $-0,6$ \\
\hline C & 0,739 & $-2,477$ & $-0,123$ & $-0,02$ & 0,08 & $-0,02$ & 6,7 & 9,0 & 9,6 & 0,3 & 2,9 & 0,6 \\
\hline $\mathrm{H}$ & 1,212 & $-4,452$ & $-0,460$ & $-0,04$ & 0,11 & 0,00 & 1,0 & 4,6 & 1,6 & $-0,5$ & $-0,4$ & 0,4 \\
\hline C & $-1,157$ & $-1,606$ & $-1,735$ & 0,02 & 0,04 & 0,07 & 7,1 & 10,5 & 8,4 & $-1,3$ & 2,5 & $-0,1$ \\
\hline $\mathrm{H}$ & $-2,091$ & $-2,946$ & $-2,987$ & 0,05 & 0,07 & 0,08 & 1,8 & 2,3 & 2,9 & 1,0 & 1,4 & 1,3 \\
\hline C & 1,849 & $-0,906$ & 1,561 & $-0,08$ & 0,01 & $-0,07$ & 6,3 & 10,8 & 8,1 & 0,7 & 2,2 & 1,1 \\
\hline $\mathrm{H}$ & 3,338 & $-1,517$ & 2,844 & $-0,10$ & 0,05 & $-0,08$ & 3,2 & 1,4 & 3,0 & $-0,8$ & 2,3 & $-0,6$ \\
\hline
\end{tabular}




\section{ADIM iterative convergence for dipole moments}

Convergence for non-polar aggregates is reached within two cycles. For covalently bonded molecules, only one cycle was done, since the proximity of the linked atoms causes some terms of the matrix product $\alpha T$ greater or equal to one and consequently, the series can diverge. However, the multiple cycles, as it is shown below, change slightly the dipole moment of the entire aggregate.

Table S26: ADIM iterative convergence of the whole pyridazine three-molecule cluster

\begin{tabular}{cccc}
\hline Cycle & $\boldsymbol{\mu}_{\mathrm{x}}$ & $\boldsymbol{\mu}_{\mathrm{y}}$ & $\boldsymbol{\mu}_{\mathrm{z}}$ \\
\hline $\mathbf{1}$ & $-0,5172$ & 2,8670 & 1,3598 \\
\hline $\mathbf{2}$ & $-0,5131$ & 2,9151 & 1,3756 \\
\hline $\mathbf{3}$ & $-0,5101$ & 2,9223 & 1,3739 \\
\hline $\mathbf{4}$ & $-0,5098$ & 2,9236 & 1,3743 \\
\hline $\mathbf{5}$ & $-0,5097$ & 2,9239 & 1,3742 \\
\hline $\mathbf{6}$ & $-0,5097$ & 2,9239 & 1,3742 \\
\hline
\end{tabular}

Table S27: ADIM iterative convergence of the whole 1,3-nitrophenol three-molecule cluster

\begin{tabular}{cccc}
\hline Cycle & $\boldsymbol{\mu}_{\mathrm{x}}$ & $\boldsymbol{\mu}_{\mathrm{y}}$ & $\boldsymbol{\mu}_{\mathrm{z}}$ \\
\hline $\mathbf{1}$ & 2,7458 & 1,9237 & $-7,8438$ \\
\hline $\mathbf{2}$ & 2,7359 & 1,9199 & $-8,1838$ \\
\hline $\mathbf{3}$ & 2,7350 & 1,9199 & $-8,2747$ \\
\hline $\mathbf{4}$ & 2,7342 & 1,9195 & $-8,2991$ \\
\hline $\mathbf{5}$ & 2,7341 & 1,9195 & $-8,3057$ \\
\hline $\mathbf{6}$ & 2,7340 & 1,9195 & $-8,3075$ \\
\hline $\mathbf{7}$ & 2,7340 & 1,9195 & $-8,3080$ \\
\hline $\mathbf{8}$ & 2,7340 & 1,9195 & $-8,3081$ \\
\hline $\mathbf{9}$ & 2,7340 & 1,9195 & $-8,3081$ \\
\hline
\end{tabular}

Table S28: ADIM iterative convergence of the whole 1,4-nitrophenol three-molecule cluster

\begin{tabular}{cccc}
\hline Cycle & $\boldsymbol{\mu}_{\mathrm{x}}$ & $\boldsymbol{\mu}_{\mathrm{y}}$ & $\boldsymbol{\mu}_{\mathrm{z}}$ \\
\hline $\mathbf{1}$ & 5,2949 & 0,5362 & 5,1836 \\
\hline $\mathbf{2}$ & 5,4745 & 0,5388 & 5,3943 \\
\hline $\mathbf{3}$ & 5,5199 & 0,5383 & 5,4474 \\
\hline $\mathbf{4}$ & 5,5312 & 0,5386 & 5,4609 \\
\hline $\mathbf{5}$ & 5,5341 & 0,5385 & 5,4643 \\
\hline $\mathbf{6}$ & 5,5349 & 0,5386 & 5,4652 \\
\hline $\mathbf{7}$ & 5,5351 & 0,5385 & 5,4654 \\
\hline $\mathbf{8}$ & 5,5351 & 0,5386 & 5,4655 \\
\hline $\mathbf{9}$ & 5,5351 & 0,5386 & 5,4655 \\
\hline
\end{tabular}




\section{Covalently bonded clusters}

Fig. S3 below shows the L-leucylglycylglycine seen from the ZY plane and the Table S29 gathers information about the $\mathrm{C}, \mathrm{N}$ and $\mathrm{O}$-atoms dipole moments that are present in the two peptide bonds. It is noticed that the biggest errors happen exactly in the direction of the resonance bonds. The 07-C14 bond is exactly in the direction of $\mathrm{X}$, and it is precisely in this direction that the greatest deviations in 07-atom occur. The same happens for the 06-C10 bond, hence the biggest errors of 06 -atom are in the $\mathrm{X}$ and $\mathrm{Z}$ directions. Regarding the $\mathrm{N}$ atoms, there is a tendency in ADIM to present higher values in module. While $\mathrm{N}$-atoms donate electronic density by resonance, it withdraws by difference of electronegativity. In the same way that the $\mathrm{O}$-atoms remove electronic density by difference of electronegativity it also receives by resonance. The purely quantum calculation tends to present higher values in module for $\mathrm{O}$-atoms if compared to ADIM.

Figure S3: L-leucylglycylglycine seen from the YZ plane

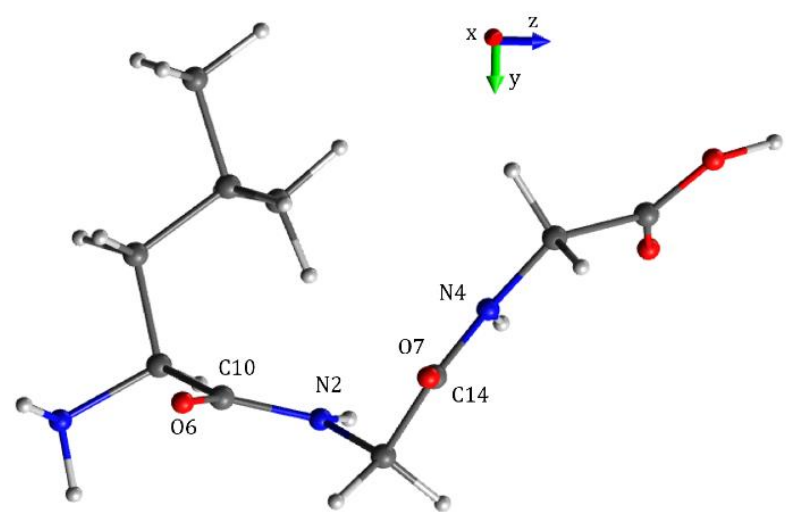

Table S29: atomic dipole moments for C, N, O-atoms within the two peptide bonds, values in a.u.

\begin{tabular}{cccc|ccc}
\hline & \multicolumn{3}{c|}{ QTAIM } & \multicolumn{3}{c}{ ADIM } \\
\hline Atom & $\boldsymbol{\mu}_{\mathrm{x}}$ & $\boldsymbol{\mu}_{\mathrm{y}}$ & $\boldsymbol{\mu}_{\mathrm{z}}$ & $\boldsymbol{\mu}_{\mathrm{x}}$ & $\boldsymbol{\mu}_{\mathrm{y}}$ & $\boldsymbol{\mu}_{\mathrm{z}}$ \\
\hline $\mathbf{N 2}$ & 0,18 & 0,19 & 0,23 & 0,19 & 0,18 & 0,39 \\
\hline $\mathbf{N 4}$ & 0,01 & $-0,13$ & 0,39 & $-0,01$ & $-0,26$ & 0,55 \\
\hline $\mathbf{0 6}$ & $-1,37$ & $-0,22$ & 0,54 & $-1,20$ & $-0,19$ & 0,40 \\
\hline $\mathbf{0 7}$ & $-1,46$ & $-0,17$ & $-0,04$ & $-1,25$ & $-0,12$ & $-0,06$ \\
\hline C10 & $-0,24$ & $-0,01$ & 0,21 & $-0,23$ & $-0,09$ & $-0,08$ \\
\hline C14 & $-0,29$ & $-0,20$ & 0,09 & $-0,18$ & $-0,02$ & $-0,10$ \\
\hline
\end{tabular}


Figure S4: Electrostatic potential maps (in u.a.) plotted over a 0.01 a.u. isodensity surface for bis(phenylethynyl)anthracene calculated from ADIM and from QTAIM. The difference between these two maps emphasizes that the largest deviations occur for covalent link

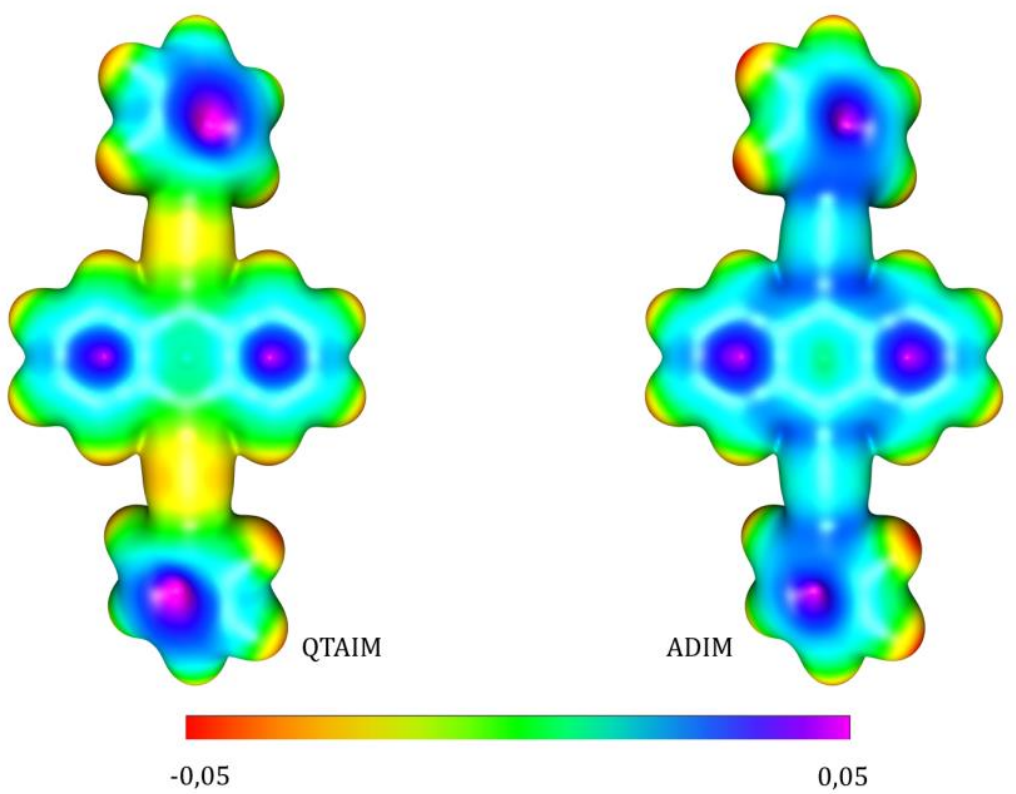

